Research Article

\title{
Vehicle State Estimation Based on Strong Tracking Central Different Kalman Filter
}

\author{
Yingjie Liu $\left(\mathbb{D},{ }^{1}\right.$ Qijiang Xu $\left(\mathbb{D},{ }^{2}\right.$ Jingxia Sun $\mathbb{D}^{\circ},{ }^{3}$ Fapeng Shen $\mathbb{D}^{3},{ }^{3}$ and Dawei Cui $\mathbb{D}^{1}$ \\ ${ }^{1}$ School of Mechanical-Electronic and Vehicle Engineering, Weifang University, Weifang 261061, China \\ ${ }^{2}$ Shandong College of Information Technology, Weifang 261061, Shandong, China \\ ${ }^{3}$ Shandong Transport Vocational College, Weifang 261206, China \\ Correspondence should be addressed to Yingjie Liu; ufoliuyingjie@163.com and Dawei Cui; wfxycdw@163.com
}

Received 11 May 2021; Revised 19 July 2021; Accepted 26 July 2021; Published 2 August 2021

Academic Editor: Yongmin Zhong

Copyright (C) 2021 Yingjie Liu et al. This is an open access article distributed under the Creative Commons Attribution License, which permits unrestricted use, distribution, and reproduction in any medium, provided the original work is properly cited.

Vehicle active safety control was a key technology to avoid serious safety accidents, and accurate acquisition of vehicle states signals was a necessary prerequisite to achieve active vehicle safety control. Based on the purpose, a 3-DOF nonlinear vehicle dynamics model containing constant noise and a nonlinear tire model were established, and several vehicle key states were estimated by a strong tracking central different Kalman filter (CDKF). The conclusion showed that the proposed estimator had higher accuracy and less computation requirement than the CKF, CDKF, and UKF estimators. Numerical simulation and experiments indicated that the proposed vehicle state estimation method not only had higher estimation accuracy but also had higher real-time function.

\section{Introduction}

Accurate and reliable state estimation is one of the necessary factors for vehicle active safety control. Some vehicle states signals cannot usually be measured directly, and the cost of direct measurement is too expensive to be widely used in the automotive industry. Therefore, vehicle states estimation has become a research hotspot in the field of vehicle active safety control.

With the development of automotive technology, there are more and more active safety devices for automobiles. It is necessary to know the longitudinal and lateral speed, yaw rate, and center of mass of the vehicle. Because it is too expensive to directly measure these parameters, the filtering method and the observer method are used to estimate the states. That is to say, states easy to measure are used to estimate states which are difficult to measure. In research on algorithms of vehicle driving state parameter estimation, most algorithms use Kalman filtering to estimate vehicle states. The advantage of Kalman filtering is that it can solve the linear minimum mean square error estimation. Since noise and interference are inevitable during the measurement process, the optimal estimation of the system can also be considered as a filtering process.

In the social situation of vigorous development of information technology, it profoundly affects the continuous development and application of the automotive technology field. And also the continuous improvement of people's life quality affects the gradual pursuit of better vehicle stability and active safety. And the vehicle's handling stability depends largely on its active safety control system. Vehicles equipped with active safety control systems can improve their handling stability and greatly reduce the incidence of traffic accidents significantly. How to obtain the driving state of the vehicle accurately and in real time has become the primary problem to be solved by the active safety control [1-5].

The problem of vehicle state estimation has been widely studied. A brief review is presented in what follows.

Sakthivel et al. [1] designed a control system to estimate vehicle state in critical situations via the extended dissipative theory. Wang et al. [6] proposed a novel combined modelbased estimation scheme to research preceding target vehicles. Lin and $\mathrm{Ha}$ [7] proposed an adaptive neuro-fuzzy 
predictor-based control (ANFPC) approach with integrated automotive radar and vehicle-to-vehicle (V2V) communication for the design of the cooperative adaptive cruise control (CACC) system which could significantly reduce the fuel consumption while ensuring driving comfort and safety. Robert et al. [8] proposed and analyzed two state estimation approaches which were Kalman filter and extended Kalman filter to estimate position, velocity, and acceleration of a vehicle. Han et al. [9] proposed and analyzed two state estimation approaches which were Kalman filter and extended Kalman filter to estimate position and velocity as well as acceleration of a vehicle. Chen et al. [3] proposed a novel probabilistic estimation method of brake pressure for electrified vehicles based on multilayer artificial neural networks (ANNs) with Levenberg-Marquardt backpropagation (LMBP) training algorithm. Kim et al. [10] proposed an interacting multiple model (IMM) approach using extended Kalman filters (EKFs) to improve multitarget state estimation performance with utilization of automotive radars. Reina et al. [11] proposed a model-based observer to assess online key motion and mass properties to increase the level of safety and driving automation. Vincent et al. [12] proposed a two-task hierarchical method named hierarchical constrained triobjective optimization (HCTO) to improve the vehicle state accuracy. Ehsan et al. [13] proposed a corner-based velocity estimation approach to estimate vehicle's traction and stability control systems. NoorA-Rahim et al. [14] proposed a Kalman filter-based UAV state estimation technique when the communication takes place over wireless links in an Internet of Things (IoT) network. Singh et al. [15] proposed an algorithm for realtime estimates of the vehicle dynamic states and tire-road contact parameters. Hamze and Ali [2] proposed the extended Kalman filter approaches in order to measure the state variables directly in an articulated heavy vehicle. Harry et al. [16] presented an alternate method for the generation and implementation of the sensor measurement variance used in an extended Kalman filter (EKF). Hamze and Ali [17] presented a novel nonlinear estimator based on state-dependent Riccati equation filter technique for state estimation of the articulated heavy vehicles. Qin et al. [18] presented a novel 2-step global sensitivity analysis algorithm to provide an in-depth sensitivity analysis of vehicle parameters on system responses with a 9 degree-of-freedom nonlinear vertical-lateral coupled vehicle model. Afzal et al. [19] presented a framework which utilized estimates of traffic flows and travel times based on real-time estimated traffic state for obtaining optimal signal timings. Wang et al. [20] proposed a vehicle side slip angle estimation method based on singular value decomposition unscented Kalman particle filtering algorithm (SVD-UPF), in order to improve the influence of particles degeneracy on the estimation of vehicle sideslip angle and ensure the nonnegative qualitative of the covariance matrix and iterative stability of the unscented Kalman filter algorithm. Liu et al. [21] presented a vehicle state estimation method based on the kernel principal component analysis and the improved Elman neural network to solve the problem that it was hard to measure some key vehicle states directly and accurately when running on the road and the cost of the measurement was high. Alexander et al. [22] presented a robust architecture in order to achieve precise tracking of the planned trajectory. Gao et al. [23] proposed a novel adaptive CKF (cubature Kalman filter) to address the problem of tightly coupled GNSS/INS integration. Gao et al. [24] presented a method of cubature rule-based distributed optimal fusion combined with identification and prediction of kinematic model error to address the problem of difficulty in achieving optimal navigation solutions in nonlinear integrated MIMU/GNSS/ CNS.

In the literature reviewed, in the existing research on vehicle state estimation, most of them focus on the improvement of estimation performance, and some focus on the reduction of estimation costs. Some researchers have not studied the problem of vehicle state estimation with hybrid algorithm. Some researchers have focused on the nonlinear system but have not proposed further improving the accuracy and robustness of estimation systems. A small number of researchers have focused on accuracy of the model but they have usually neglected the noise influence.

Traditional Kalman filtering algorithms such as extended Kalman filtering and unscented Kalman filtering use recursive iterations, which can simplify calculations and obtain relatively accurate estimation results to implement. Therefore, the Kalman filtering algorithm has become one of the algorithms commonly used by researchers. In the process of the unscented Kalman filtering algorithm estimating vehicle state variables, the noise covariance matrices $Q$ and $R$ are constant matrices. And the measurement noise covariance matrix is an important parameter in the filtering process. If $R$ is too large or too small, the filtering effect will become worse. And also $R$ with large error value may cause the algorithm to diverge. After compensation, the system noise has a certain degree of robustness, so the estimation of $Q$ is of practical significance. The measurement noise is mainly caused by external interference, which has a large uncertainty.

The strong tracking CDKF uses the orthogonality principle to compel the output residuals to be orthogonal or approximately orthogonal by introducing a fading factor into the state prediction covariance matrix, which has the ability of tracking the sudden changing of the system. At the same time, the strong tracking CDKF uses a central difference transform to approximate the posterior mean and covariance of the state of the nonlinear Gaussian system, without the need of calculating a complex Jacobean matrix. The process can improve the solving efficiency and filtering accuracy.

Aimed on the reasons mentioned above, the paper proposes the strong tracking CDKF algorithm to estimate the vehicle key states. In the rest of the paper: Section 2 presents the mathematical Model. Section 3 provides the strong tracking CDKF algorithm. Sections 4 and 5 present numerical simulation, experimental verification, and conclusions of the paper, respectively. 


\section{Mathematical Model of Vehicle State Estimation Problem}

2.1. 3-DOF Vehicle Model. The vehicle state estimation model is established based on a 3-DOF vehicle model. The 3DOF vehicle model is shown in Figure 1xoyis the vehicle coordinate system, and the origin of the vehicle coordinate system coincides with the center of mass of the vehicle.

The 3-DOF vehicle model ignores the movement of the vehicle in the roll, pitch, and vertical directions, and only considers the movement in the longitudinal, lateral, and yaw directions. It is assumed that the mechanical characteristics of each tire are the same. According to Newton's second law, the force balance equations along the $x$-axis, $y$-axis, and $z$ axis can be obtained.

In the $x$-axis direction,

$$
a_{x}=\dot{v}_{x}-v_{y} r .
$$

In the $y$-axis direction,

$$
a_{y}=\dot{v}_{y}+v_{x} r .
$$

Around the $z$-axis,

$$
I_{z} \dot{r}_{x}=\sum M
$$

The side slip angle of the center of mass is

$$
\beta=\arctan \left(\frac{v_{y}}{v_{x}}\right) \text {. }
$$

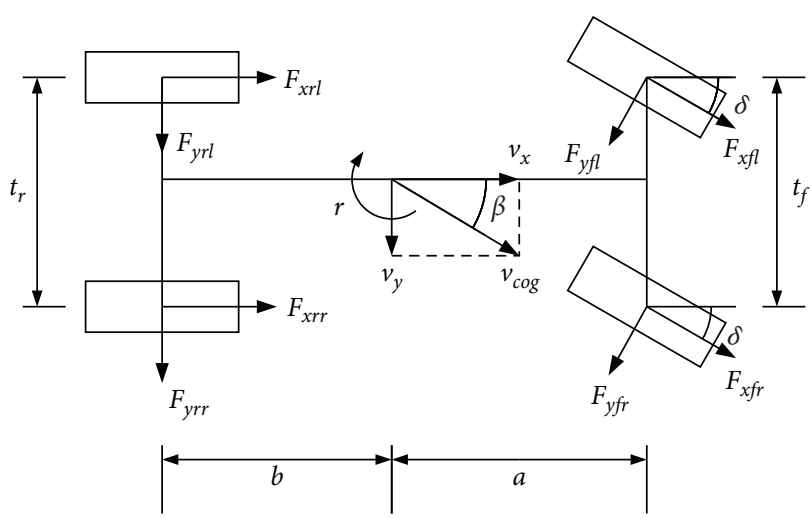

FIGURE 1: 3-DOF vehicle model.

Based on the differential equation of vehicle motion, the side slip angle of each wheel can be obtained as

$$
\left\{\begin{array}{l}
\alpha_{f l, f r}=\delta-\operatorname{arctg} \frac{v_{y}+a r}{v_{x} \pm\left(t_{f} / 2\right) r}, \\
\alpha_{r l, r r}=-\operatorname{arctg} \frac{v_{y}-b r}{v_{x} \pm\left(t_{r} / 2\right) r}
\end{array}\right.
$$

where $t_{f}$ is the front track.

According to equations (1) to (5), the dynamic equations of longitudinal, lateral, and yaw directions are further obtained as

$\left\{\begin{array}{l}\sum F_{x}=m a_{x}=\left(F_{x f l}+F_{x f r}\right) \cos (\delta)+F_{x r l}+F_{x r r}-\left(F_{y f l}+F_{y f r}\right) \sin (\delta), \\ \sum F_{y}=m a_{y}=\left(F_{x f l}+F_{x f r}\right) \sin (\delta)+F_{y r l}+F_{y r r}+\left(F_{y f l}+F_{y f r}\right) \cos (\delta), \\ \sum M_{z}=I_{z} \dot{r}=\left(F_{x f l}+F_{x f r}\right) a \sin (\delta)+\left(F_{y f l}+F_{y f r}\right) a \cos (\delta)-\left(F_{y r l}+F_{y r r}\right) b-\left(F_{x f l}-F_{x f r}\right) \frac{t_{f}}{2} \cos (\delta)-\left(F_{x r l}-F_{x r r}\right) \frac{t_{f}}{2}+\left(F_{y f l}-F_{y f r}\right) \frac{t_{f}}{2} \sin (\delta),\end{array}\right.$

where $m$ is the vehicle mass; $v_{x}$ and $v_{y}$ are the longitudinal and lateral speed, respectively; $a_{x}$ and $a_{y}$ are the longitudinal and lateral acceleration, respectively; $r$ is the yaw rate of the vehicle; $\beta$ is the side slip angle; $I_{z}$ is the moment of inertia around the $z$-axis; $a$ and $b$ are the distances of front and rear axle from the center of gravity, respectively; $\delta$ is the front steering angle; $F_{x i j}$ and $F_{y i j}$ are the longitudinal and lateral forces of the tire, respectively; and $M_{z}$ is the yaw moment.

2.2. Modified "Fiala Model". The force between the tire and the road surface (longitudinal and lateral force) has an important impact on the driving safety of the vehicle. And the tire force is related to factors such as load, tire pressure, and road adhesion coefficient. When the tire is in a nonlinear state, it is difficult to establish a more accurate theoretical model to describe it. The "Magic formula" tire model can more accurately fit the nonlinear characteristics of the tire. The "Magic formula" tire model is selected here to describe the dynamic characteristics of the tire. The general expression of the "Magic formula" is [25]

$$
y=D \sin \{C \arctan [B x-E(B x-\arctan (B x))]\}+S,
$$

where $x$ is the side slip angle of the tire and $y$ is the tire force or torque. The parameters $B, C, D, E$, and $S$ are related to the tire load, wheel camber angle, and adhesion conditions of road surface. Different tire load, wheel camber angle, and road surface adhesion conditions correspond to different coefficients. 
2.3. Nonlinear Vehicle System Containing Noise. The state vector of the nonlinear vehicle system is set as

$$
\mathbf{x}=\left[v_{x}, r, a_{y}, \beta,\right]^{T} .
$$

The system input is $\mathbf{u}=\left[\delta, a_{x}\right]^{T}$, and the observation vector is

$$
\mathbf{y}=\left[a_{y}, r\right]
$$

\section{Strong Tracking CDKF}

The specific calculation steps of the algorithm are as follows:

3.1. Equation Predicting. According to the symmetric sampling principle, the statistical characteristics $\left(\widehat{x}_{k}, \mathbf{P}_{k}\right)$ of the state vector $\mathbf{x}$ at time $k$ are used to calculate the Sigma point $\xi_{i, k}$ and its weight coefficients $W_{i}^{m}, W_{i}^{c 1}$, and $W_{i}^{c 2}$ :

$$
\left\{\begin{array} { l } 
{ \xi _ { 0 , k } = \hat { x } _ { k } , } \\
{ \xi _ { i , k } = \hat { x } _ { k } + ( h \sqrt { \mathbf { P } _ { k } } ) _ { i } , } \\
{ \xi _ { i + n , k } = \hat { x } _ { k } - ( h \sqrt { \mathbf { P } _ { k } } ) _ { i } , }
\end{array} \left\{\begin{array}{l}
W_{0}^{m}=\frac{h^{2}-n}{h^{2}}, \\
W_{i}^{m}=\frac{1}{2 h^{2}}, \\
W_{i}^{c 1}=\frac{1}{4 h^{2}}, \\
W_{i}^{c 2}=\frac{h^{2}-1}{4 h^{2}}
\end{array}\right.\right.
$$

where $h$ is the length of the center difference interval and $h^{2} \geq 1 ; i=1,2, \ldots, n$.

$\xi_{i, k}$ is transformed into $\gamma_{i, k+1 \mid k}$ which is used to calculate the state prediction $\hat{x}_{k+1 \mid k}$ and covariance matrix $\mathbf{P}_{k+1 \mid k}^{(l)}$ by nonlinear state function.

$$
\begin{aligned}
\gamma_{i, k+1 \mid k} & =f\left(\xi_{i, k}, u_{k}\right), \quad i-0,1, \ldots, 2 n, \\
\widehat{x}_{k+1 \mid k} & =\sum_{i=0}^{2 n} W_{i}^{m} \gamma_{i, k+1 \mid k}, \\
\mathbf{P}_{k+1 \mid k}^{(l)} & =\sum_{i=1}^{n}\left[W_{i}^{c 1}\left(\gamma_{i, k+1 \mid k}-\gamma_{i+n, k+1 \mid k}\right)^{2}+W_{i}^{c 2}\left(\gamma_{i, k+1 \mid k}+\gamma_{i+n, k+1 \mid k}-2 \gamma_{0, k+1 \mid k}\right)^{2}\right]+Q .
\end{aligned}
$$

In order to make the filter robust to the uncertainties of the system model, the fading factor matrix $\lambda_{k+1}$ is introduced in the state prediction covariance matrix $\mathbf{P}_{k+1 \mid k}^{(l)}$ to obtain equation (12).

$$
\mathbf{P}_{k+1 \mid k}=\lambda_{k+1} \sum_{i=1}^{n}\left[W_{i}^{c 1}\left(\gamma_{i, k+1 \mid k}-\gamma_{i+n, k+1 \mid k}\right)^{2}+W_{i}^{c 2}\left(\gamma_{i, k+1 \mid k}+\gamma_{i+n, k+1 \mid k}-2 \gamma_{0, k+1 \mid k}\right)^{2}\right]+Q .
$$

The fading factor matrix $\lambda_{k+1}$ is determined as

$$
\lambda_{k+1}=\left(\begin{array}{l}
\mathbf{C}_{k+1}, \mathbf{C}_{k+1}>1 \\
\mathbf{I}_{n \times n}, \mathbf{C}_{k+1} \leq 1
\end{array}\right.
$$

where

$$
\begin{aligned}
& \mathbf{C}_{k+1}=\frac{\operatorname{tr}\left[\mathbf{N}_{k+1}\right]}{\operatorname{tr}\left[\mathbf{M}_{k+1}\right]}, \\
& \mathbf{N}_{k+1}=\mathbf{V}_{k+1}-\mathbf{R}-\left[\mathbf{P}_{\tilde{x}_{k+1}^{(l)}}^{(l)}\right]^{T}\left[\mathbf{P}_{k+1 \mid k}^{(l)}\right]^{-1} \mathrm{Q}\left[\mathbf{P}_{k+1 \mid k}^{(l)}\right]^{-1} \mathbf{P}_{\widetilde{x}_{k+1}}^{(l)} \tilde{z}_{k+1}, \\
& \mathbf{M}_{k+1}=\left[\mathbf{P}_{k+1 \mid k}^{(l)}-Q\right]\left[\mathbf{P}_{k+1 \mid k}^{(l)}\right]^{-1} \mathbf{P}_{\widetilde{x}_{k+1}^{(l)}}^{(l)} \tilde{z}_{k+1}\left[\mathbf{P}_{\widetilde{x}_{k+1}}^{(l)} \tilde{z}_{k+1}\right]^{T}\left[\mathbf{P}_{k+1 \mid k}^{(l)}\right]^{-1},
\end{aligned}
$$

where $\operatorname{tr}(\cdot)$ is the operator of matrix trace, $\mathbf{P}_{\widetilde{z}_{k+1}}^{(l)}$ and $\mathbf{P}_{\widetilde{x}_{k+1}}^{(l)} \widetilde{z}_{k+1}$ are subcovariance matrix and cross-covariance matrix without fading factors, respectively. $V_{k+1}$ is the covariance matrix of the actual output residual sequence which is determined by the following method:

$$
\mathbf{V}_{k+1}=\left(\begin{array}{ll}
\varepsilon_{1} \varepsilon_{1}^{T}, & k=0, \\
\frac{\sigma \mathbf{V}_{k}+\varepsilon_{k+1} \varepsilon_{k+1}^{T}}{1+\sigma}, & k \geq 1,
\end{array} \boldsymbol{\varepsilon}_{k+1}=z_{k+1}-\widehat{z}_{k+1 \mid k},\right.
$$

where $0<\sigma \leq 1$ is the forgetting factor which is usually set as $\sigma=0.95$.

$$
\mathbf{P}_{\widetilde{z}_{k+1}}^{(l)} \text { and } \mathbf{P}_{\widetilde{x}_{k+1}}^{(l)} \widetilde{z}_{k+1} \text { can be obtained by } \widehat{x}_{k+1 \mid k} \text { and } \mathbf{P}_{k+1 \mid k}^{(l)} \text { : }
$$




$$
\left\{\begin{aligned}
\xi_{0, k+1 \mid k}^{(l)}= & \widehat{x}_{k+1 \mid k} \\
\xi_{i, k+1 \mid k}^{(l)}= & \widehat{x}_{k+1 \mid k}+\left(h \sqrt{\mathbf{P}_{k+1 \mid k}^{(l)}}\right), \quad i=1,2, \ldots, n, \\
\xi_{i+n, k+1 \mid k}^{(l)} & =\widehat{x}_{k+1 \mid k}-\left(h \sqrt{\mathbf{P}_{k+1 \mid k}^{(l)}}\right)_{i} \\
\chi_{i, k+1 \mid k}^{(l)} & =h\left(\xi_{i, k+1 \mid k}^{(l)}\right), \quad i=0,1, \ldots, 2 n \\
\widehat{z}_{k+1 \mid k} & =\sum_{i=0}^{2 n} W_{i}^{m} \chi_{i, k+1 \mid k}^{(l)}, \\
\mathbf{P}_{\widetilde{z}_{k+1}^{(l)}} & =\sum_{i=1}^{n}\left[W_{i}^{c 1}\left(\chi_{i, k+1 \mid k}^{(l)}-\chi_{i+n, k+1 \mid k}^{(l)}\right)^{2}+W_{i}^{c 2}\left(\chi_{i, k+1 \mid k}^{(l)}+\chi_{i+n, k+1 \mid k}^{(l)}-2 \chi_{0, k+1 \mid k}^{(l)}\right)^{2}\right]+\mathbf{R} \\
\mathbf{P}_{\widetilde{x}_{k+1}^{(l)} \tilde{z}_{k+1}} & =\sum_{i=1}^{n} \sqrt{W_{i}^{c 1}}\left[\left(\xi_{i, k+1 \mid k}^{(l)}-\widehat{x}_{k+1 \mid k}\right)\left(\chi_{i, k+1 \mid k}^{(l)}-\chi_{i+n, k+1 \mid k}^{(l)}\right)^{T}\right] .
\end{aligned}\right.
$$

According to the symmetric sampling strategy, the statistical characteristics of the state vector prediction are used to calculate the Sigma point:

$$
\left\{\begin{array}{l}
\xi_{0, k+1 \mid k}=\widehat{x}_{k+1 \mid k}, \\
\xi_{i, k+1 \mid k}=\widehat{x}_{k+1 \mid k}+\left(h \sqrt{\mathbf{P}_{k+1 \mid k}}\right)_{i}, \quad i=1,2, \ldots, n, \\
\xi_{i+n, k+1 \mid k}=\widehat{x}_{k+1 \mid k}-\left(h \sqrt{\mathbf{P}_{k+1 \mid k}}\right)_{i}
\end{array}\right.
$$

$\xi_{i, k+1 \mid k}$ is transformed into $\chi_{i, k+1 \mid k}$ which is used to calculate the self-covariance matrix $\mathbf{P}_{\widetilde{z}_{k+1}}$ and the cross-covariance matrix $\mathbf{P}_{\tilde{x}_{k+1}} \tilde{z}_{k+1}$ through nonlinear measurement function, after introducing the fading factor:

$$
\begin{aligned}
\boldsymbol{\chi}_{i, k+1 \mid k} & =h\left(\xi_{i, k+1 \mid k}\right), \quad i=0,1, \ldots, 2 n, \\
\mathbf{P}_{\widetilde{z}_{k+1}} & =\sum_{i=1}^{n}\left[W_{i}^{c 1}\left(\boldsymbol{\chi}_{i, k+1 \mid k}-\boldsymbol{\chi}_{i+n, k+1 \mid k}\right)^{2}+W_{i}^{c 2}\left(\boldsymbol{\chi}_{i, k+1 \mid k}+\boldsymbol{\chi}_{i+n, k+1 \mid k}-2 \boldsymbol{\chi}_{0, k+1 \mid k}\right)^{2}\right]+\mathbf{R}, \\
\mathbf{P}_{\widetilde{x}_{k+1} \tilde{z}_{k+1}} & =\sum_{i=1}^{n} \sqrt{W_{i}^{c 1}}\left[\left(\xi_{i, k+1 \mid k}-\widehat{x}_{k+1 \mid k}\right)\left(\boldsymbol{\chi}_{i, k+1 \mid k}-\boldsymbol{\chi}_{i+n, k+1 \mid k}\right)^{T}\right] .
\end{aligned}
$$

3.2. Measurement Updating. After obtaining the new measurement $\mathbf{z}_{k+1}$, a strong tracking CDKF filter updating is performed: $\widehat{x}_{k+1}=\widehat{x}_{k+1 \mid k}+\mathrm{K}_{k+1}\left(z_{k+1}-\widehat{z}_{k+1 \mid k}\right)$

$$
\begin{aligned}
& \mathbf{K}_{k+1}=\mathbf{P}_{\widetilde{x}_{k+1} \tilde{z}_{k+1}} \mathbf{P}_{\tilde{z}_{k+1}^{-1}}^{-1}, \\
& \mathbf{P}_{k+1}=\mathbf{P}_{k+1 \mid k}-\mathbf{K}_{k+1} \mathbf{P}_{\widetilde{z}_{k+1}} \mathbf{K}_{k+1}^{T} .
\end{aligned}
$$

According to the expression of the strong tracking filtering (STF) and strong tracking CDKF as well as reference [26], it is not difficult to see that the application of central difference transformation with more accurate calculating the posterior mean and covariance of the state can not only overcome the shortcomings of low first-order approximation accuracy of the STF but also avoid the trouble of calculating the Jacobian matrix. Strong tracking CDKF does not require derivative operations, and its numerical stability and estimation accuracy are significantly improved compared to STF. In addition, strong tracking CDKF inherits the advantage of strong robustness of the STF when the system model is uncertain by introducing a fading factor into the state covariance matrix.

\section{Numerical Simulation and Experimental Verification}

4.1. Numerical Simulation. In order to verify the feasibility and accuracy of the strong tracking CDKF algorithm for vehicle state and parameter estimation, the vehicle dynamics simulation software CarSim in the Matlab/Simulink environment is used for simulation. CarSim is a software product of mechanical simulation company that simulates vehicle behavior. It can carry out the interaction between threedimension dynamic vehicle response, advanced controller, driver control, and three-dimension road. It can be easily used by most engineers and technicians. Each package includes VS browser (GUI and database management), VS 
visualizer (animation and drawing), online help, and VS solver for detailed mathematical models. The mathematical model can be run alone, or by the third-party simulation software, such as Simulink, Lab VIEW, and so on. And also, the CarSim is a simulation software used in the automotive industry. The software is essentially to establish a vehicle model first, then set the parameters according to its own simulation contents, and display the simulation results through 3D animation or table data after the processor calculation. The CarSim software system can perform simulations with much other software. For example, CarSim and Simulink can perform simulation together. When implementing simulation, the required variables from various variables in Simulink are selected and imported into CarSim. The variables include vehicle control input, tire force and torque, spring and damping force, steering system drive angle, driveline torque, brake torque, brake pressure, and so on. The data after modeling and simulation can also be exported to other simulation software as a data source for simulation and data analysis.

The double lane change road test and the slalom road test are the basic working conditions in vehicle stability evaluation, which are widely used in the development and verification of stability control system and belong to closed-loop test.

As the standard test methods for vehicle stability control, the double lane change road and the slalom road operating conditions have good objectivity and repeatability. The purpose of vehicle state parameter estimation in this paper is to provide a basis for subsequent vehicle stability control. So the double lane change road and the slalom road operating conditions are chose as the simulation verification conditions.

When simulations are carried out using the software, the test speed is many times faster than the actual test speed. The software can be used to simulate the feedback made by the vehicle under various inputs such as the driver and the ground conditions. And also, it can be used to help improving the performances of stability, braking, ride comfort, power, and economy of the vehicle.

Nowadays, CarSim has gradually been applied by more R\&D personnel with its own advantages.

In order to verify the effectiveness of the proposed strong tracking CDKF algorithm, double lane change road, and slalom road are used to analyze and compare the unscented Kalman filter and the strong tracking CDKF. And CarSim is proposed to perform a virtual test.

The calculation parameters are shown in Table 1 [27].

4.1.1. Double Lane Change Test Road. Figure 2 shows the lateral displacement and vertical displacement of the center of mass when the vehicle moves through the double lane change test road at a speed of $80 \mathrm{~km} / \mathrm{h}$.

Observational measurements with noise interference are shown in Figure 3.

Figure 4 shows the estimated value of the state variables. It can be seen from Figure 4 that the estimated values of the four states which are the lateral velocity, the lateral acceleration, the yaw rate, and the side slip angle are in good
TABLE 1: Simulation parameters.

\begin{tabular}{lc}
\hline Parameter & Value \\
\hline $\mathrm{M}(\mathrm{kg})$ & 1528 \\
$\left.I_{\mathrm{z}}(\mathrm{kg} \mathrm{m})^{2}\right)$ & 2440 \\
$a(\mathrm{~m})$ & 1.48 \\
$b(\mathrm{~m})$ & 1.08 \\
$I$ & 26.7 \\
\hline
\end{tabular}

agreement with the virtual test values at $80 \mathrm{~km} / \mathrm{h}$. And the follow ability of the lateral acceleration and yaw rate are slightly worse at the peaks and troughs.

With the improvement of road conditions and the development of automobile technology, it is very common for vehicles to travel at speeds above $100 \mathrm{~km} / \mathrm{h}$, and in general, emergency situations for vehicles also occur under highspeed driving conditions. Therefore, the vehicle dynamic control system and the design of the estimator should take into account the operating conditions at higher speeds.

Figure 5 shows the lateral displacement and vertical displacement of the center of mass when the vehicle moves through the double lane change test road at a speed of $120 \mathrm{~km} / \mathrm{h}$.

It can be seen from Figure 5 that as the vehicle speed increases, the longitudinal displacement of the double lane change road completed in the same time increases.

From the degree of agreement between the estimated value and the virtual test value in Figure 6, it can be seen that generally speaking, the vehicle speed still has better estimation accuracy. At the same time, the lateral velocity and the slip angle are significantly increased, and the estimated value of these two state variables does not match the virtual test value as well as the low-speed situation. Especially the estimation accuracy at the peak and trough positions is not good. This is because the state changes drastically at high speeds, resulting in poor follow-up of the estimated value. In addition, relative to low-speed conditions, lateral acceleration and yaw rate still have high estimation accuracy.

4.1.2. Slalom Road. Figure 7 shows the lateral displacement and longitudinal displacement of the center of mass when the vehicle moves through the slalom road at a speed of $80 \mathrm{~km} / \mathrm{h}$.

Observational measurements with noise interference are shown in Figure 8.

From Figure 9, it can be seen that when the vehicle is driving at a speed of $80 \mathrm{~km} / \mathrm{h}$, the maximum lateral acceleration has reached at $8 \mathrm{~m} / \mathrm{s}^{2}$, and the tire has entered the nonlinear region. Generally speaking, the estimated values of the four states are in good agreement with the virtual test values. The estimated values of each state have larger errors on the peaks and troughs compared with the virtual test values.

Figure 10 shows the lateral displacement and longitudinal displacement of the center of mass when the vehicle moves through the slalom road at a speed of $120 \mathrm{~km} / \mathrm{h}$.

It can be seen from Figure 10 that as the vehicle speed increases, the longitudinal displacement of the slalom road completed in the same time increases. 


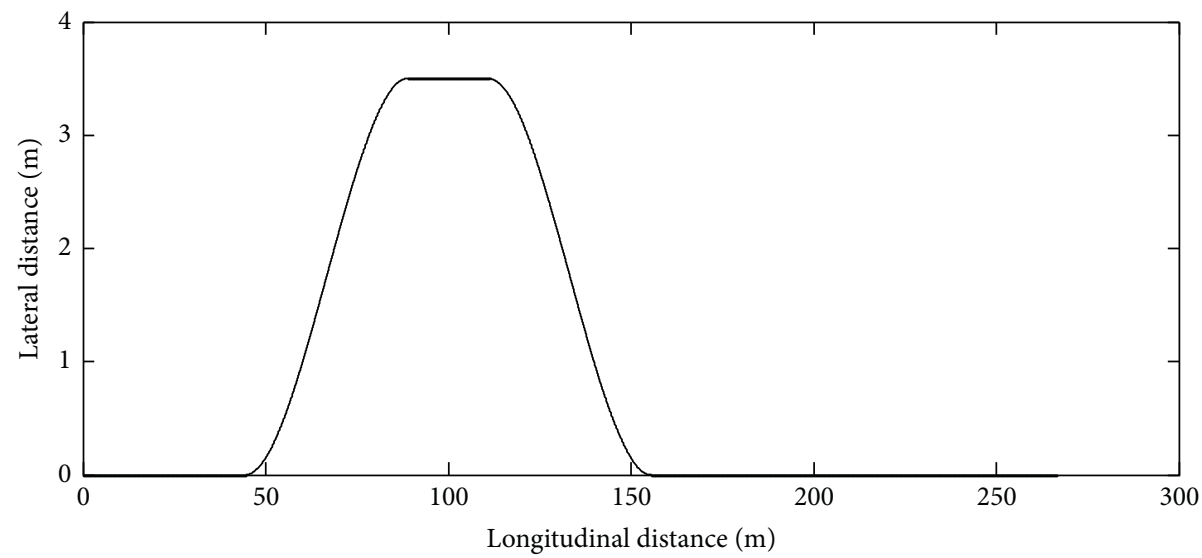

Figure 2: Lateral distance of double lane change road at $80 \mathrm{~km} / \mathrm{h}$.

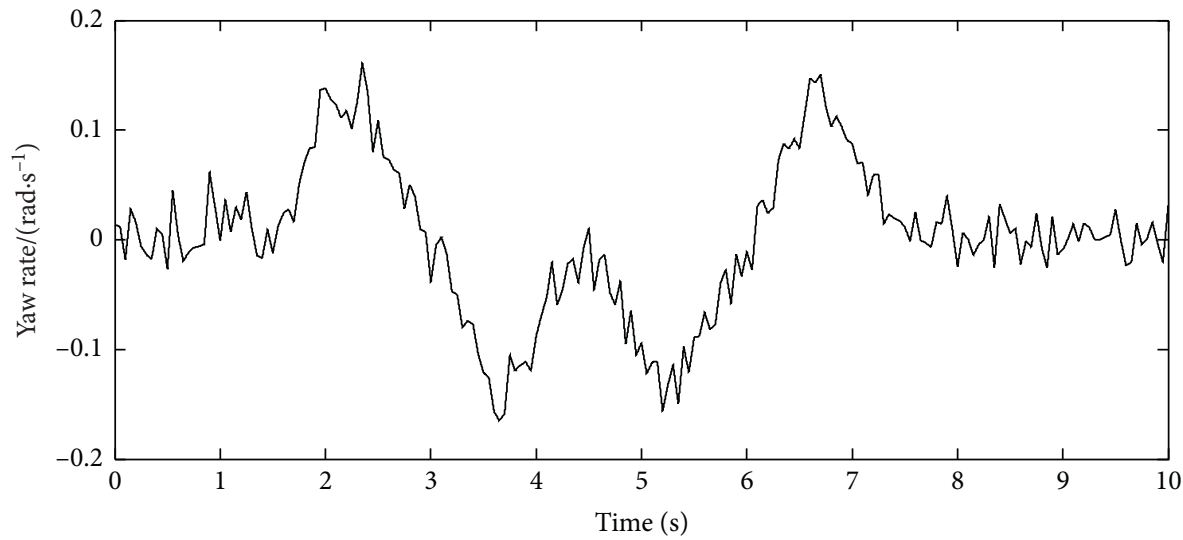

(a)

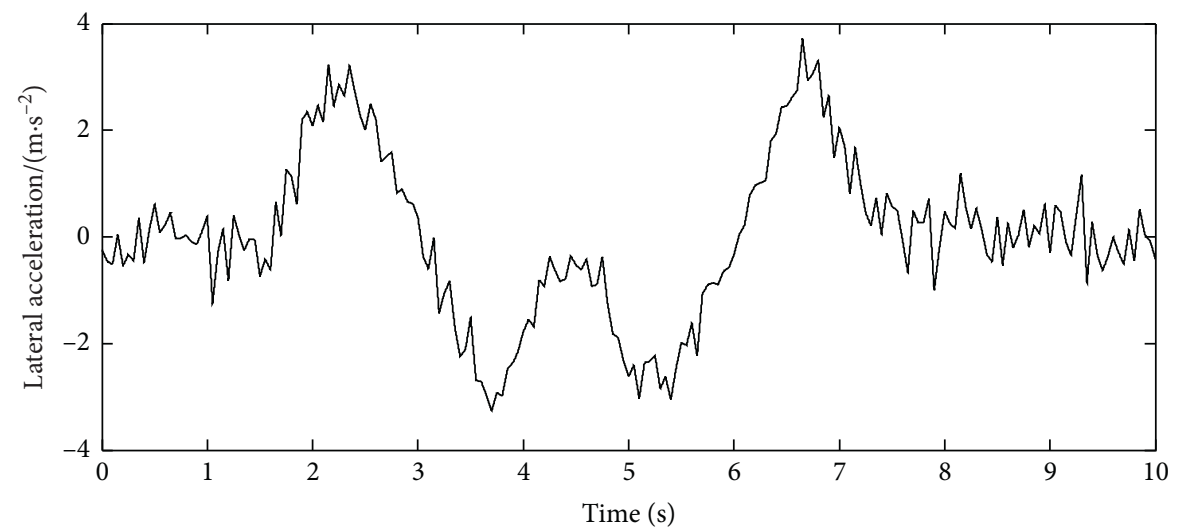

(b)

Figure 3: Observational measurements (double lane change road): (a) observation measurements of the yaw rate and (b) observational measurements of the lateral acceleration.

From Figures 11(a) and 11(b), it can be seen that the lateral speed of the vehicle has increased significantly with the increases of the vehicle longitudinal speed, but the lateral acceleration has not changed much. The former reflects that under the condition of constant lateral displacement, the sideslip tendency of the vehicle increases after the vehicle speed increases. It can be seen from the figure that the estimation accuracy of the strong tracking CDKF algorithm for the lateral speed at this vehicle speed is slightly lower than that of the low-speed situation. The latter reflects that the lateral force of the tire does not change much at the two vehicle speeds. It can be seen from the figure that the estimation accuracy of the lateral acceleration is not greatly affected by the vehicle speed.

From Figure 11(c), it can be seen that the yaw rate of the vehicle is reduced at a higher speed, which shows that the 


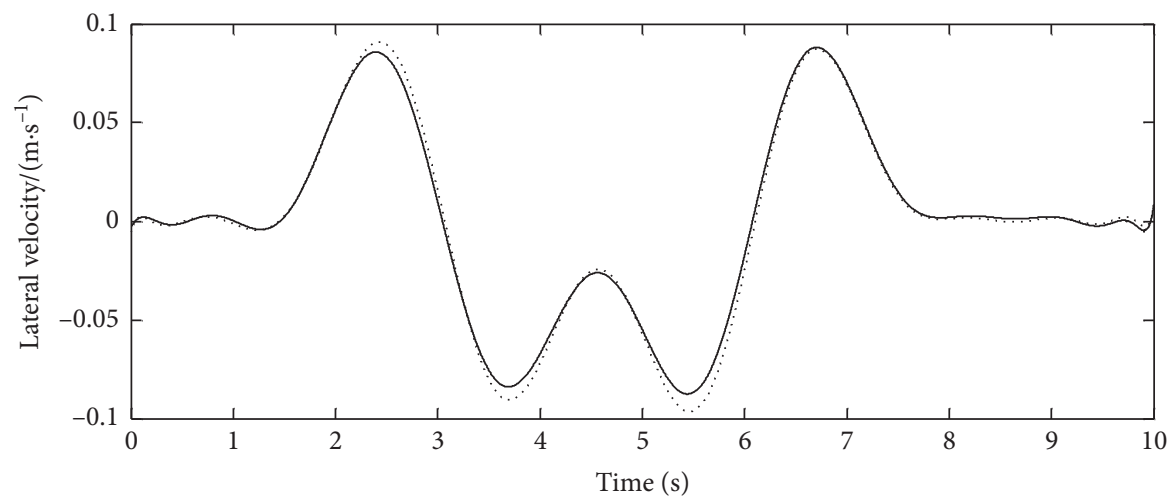

CarSim

__ Strong tracking CDKF

(a)

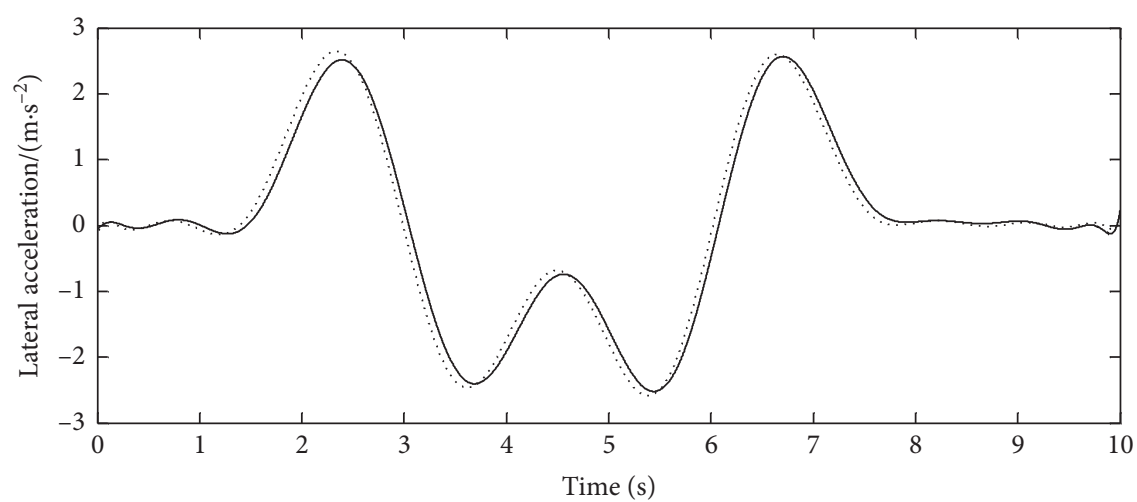

... CarSim

_ _ Strong tracking CDKF

(b)

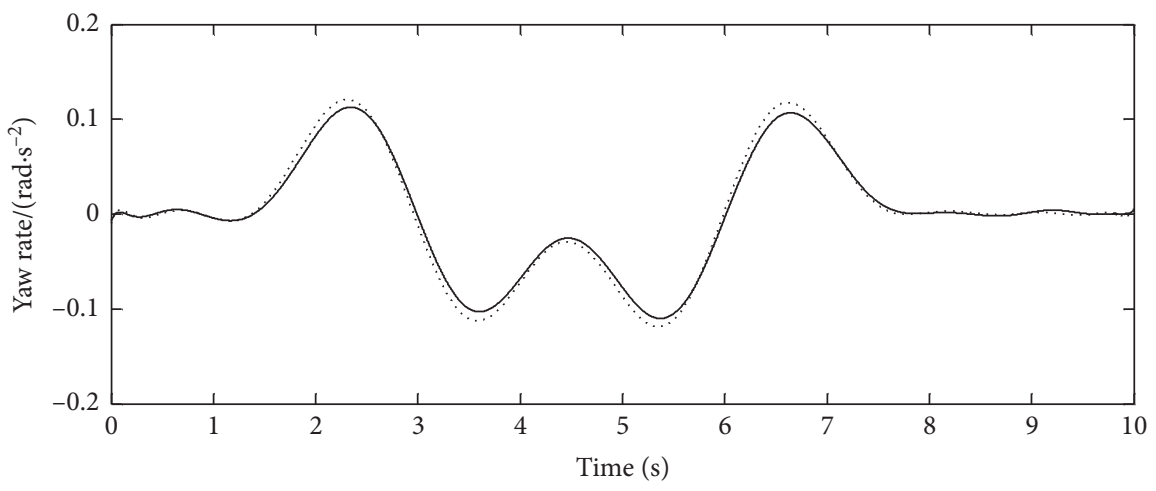

CarSim

Strong tracking CDKF

(c)

Figure 4: Continued. 


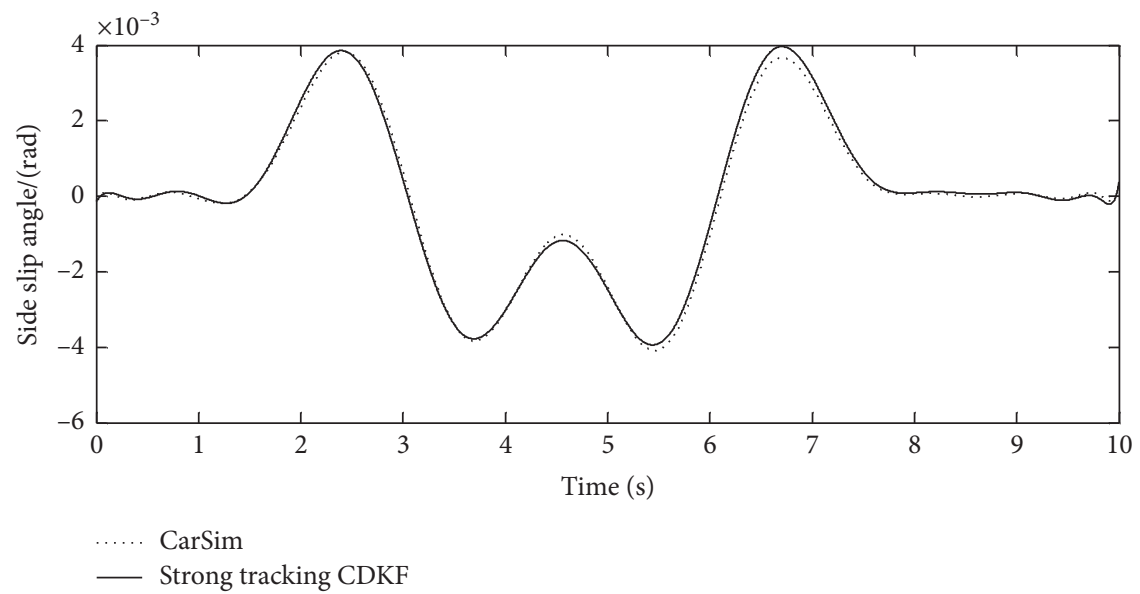

(d)

Figure 4: Estimated value of the state variables at $80 \mathrm{~km} / \mathrm{h}$ (double lane change road): (a) estimated value of the lateral velocity, (b) estimated value of the lateral acceleration, (c) estimated value of the yaw rate, and (d) estimated value of the side slip angle.

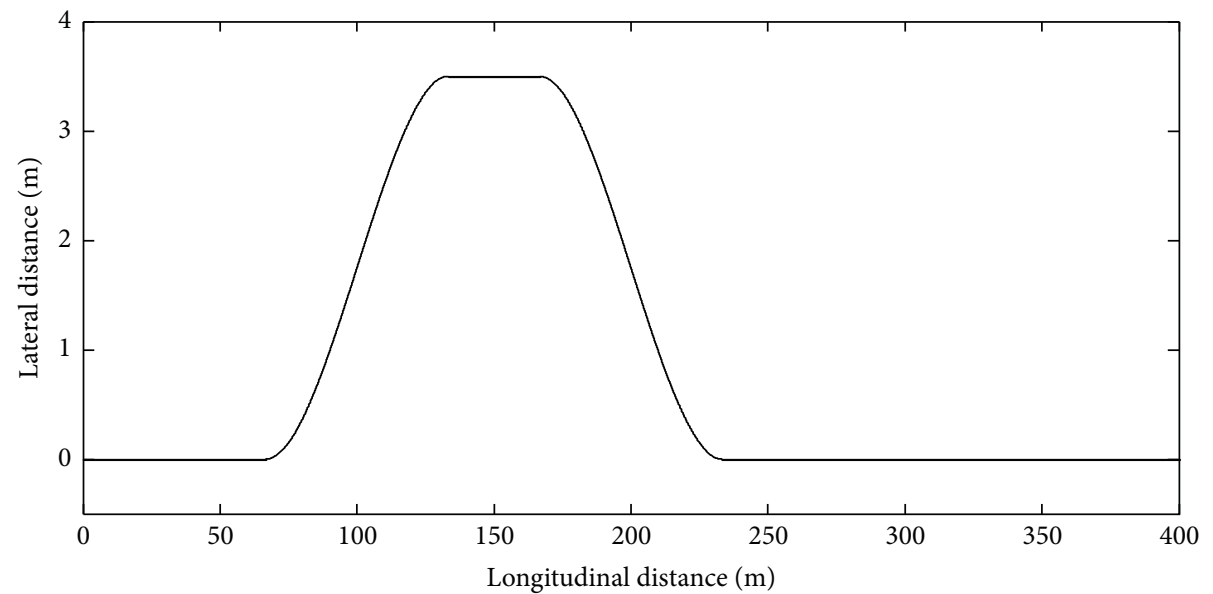

FIGURE 5: Lateral distance of double lane change road at $120 \mathrm{~km} / \mathrm{h}$.

yaw moment of the vehicle reduces. The degree of agreement between the estimated value and the test values is better when the speed is lower. From Figure 11(d), it can be seen that the amplitude of the slip angle reduces at higher speeds. This is because the increase in lateral speed is not as large as the increase in longitudinal speed after the vehicle speed increases. The estimation accuracy is roughly equivalent to that of the low speed.

The estimation effects of the two algorithms (strong tracking $\mathrm{CDKF}, \mathrm{UKF}$ ) are compared under condition of tracking slalom road with $120 \mathrm{~km} / \mathrm{h}$. Figure 12 is the estimation value of the four key states.

It can be seen from Figure 12 that the estimated values of the four algorithms are very close. And also, the simulation shows that the proposed algorithm of the paper has higher precision and stronger ability to solve the problem of vehicle state estimation.

The error indicators which are the average absolute error (MAE) and root mean square error (RMSE) of the four estimation algorithms are given in Table 2. And the expressions of the two error indicators are as follows:

$$
\begin{aligned}
\operatorname{MAE}(x) & =\frac{\sum_{k=1}^{N}\left|x_{k \mid k}-x_{\text {actual }}(k)\right|}{N}, \\
\operatorname{RMSE}(x) & =\sqrt{\frac{\sum_{k=1}^{N}\left(x_{k \mid k}-x_{\text {actual }}(k)\right)^{2}}{N}},
\end{aligned}
$$

where $x_{k \mid k}$ and $x_{\text {actual }}(k)$ are the estimated value and the virtual test value at time $k$, respectively.

It can be seen from Table 2 that, compared with the CKF, $\mathrm{CDKF}$, and the UKF algorithms, the strong tracking CDKF algorithm has higher estimation accuracy under the same simulation conditions, and the peak relative error is smaller. And also the simulation results indicate that the proposed algorithm has a high degree of agreement of the tracking trend. So the accuracy of the strong tracking CDKF is higher than that of the CKF, CDKF, and the UKF in the vehicle estimation.

4.2. Experimental Verification. A real vehicle test of tracking double lane change road is carried out, and the test is performed in accordance with ISO/TR3888-2004 to obtain 


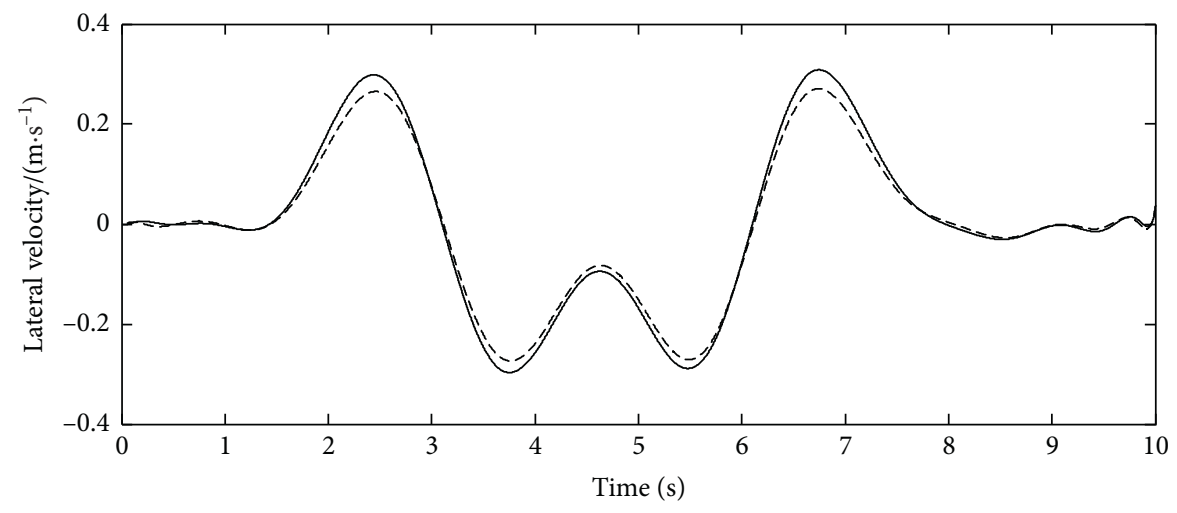

- - - CarSim

_ Strong tracking CDKF

(a)

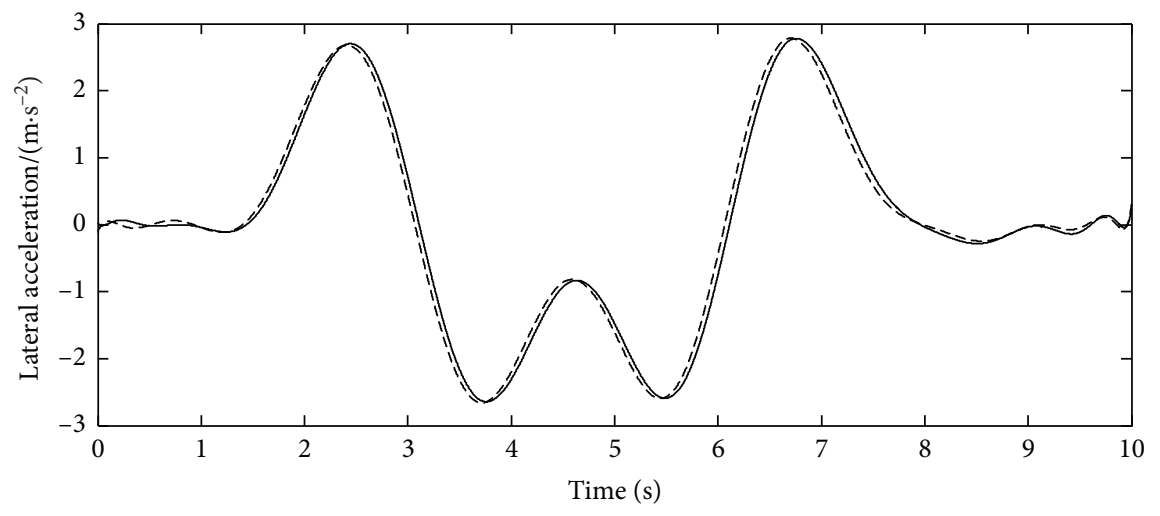

- - - CarSim

— Strong tracking CDKF

(b)

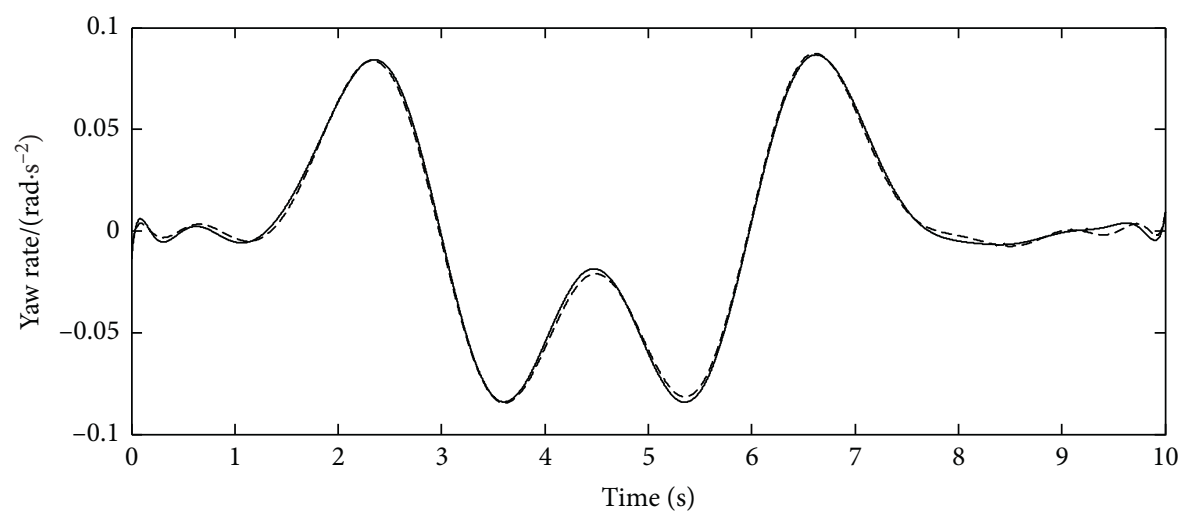

CarSim

— Strong tracking CDKF

(c)

FIgURE 6: Continued. 


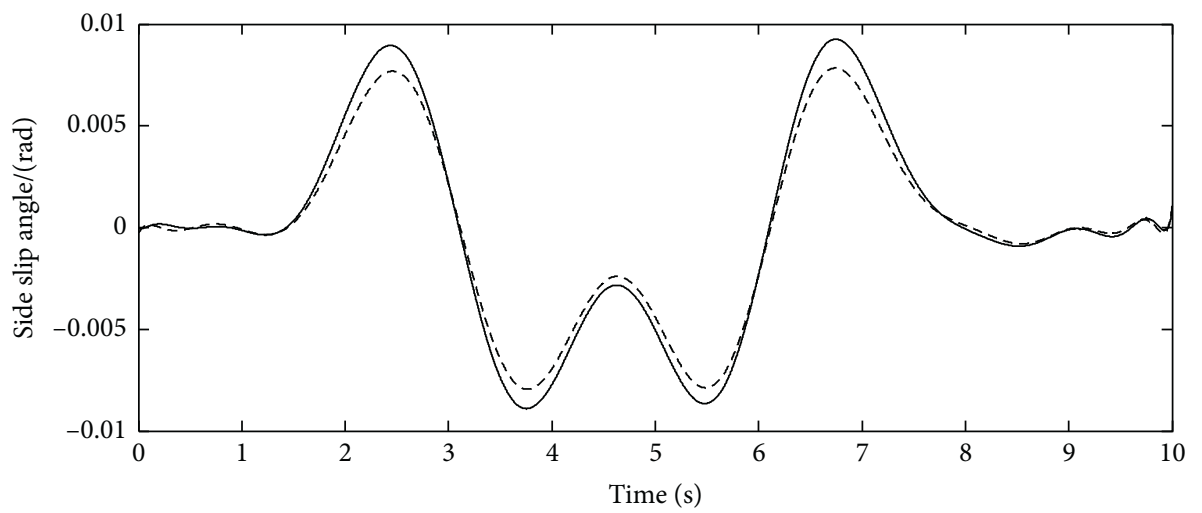

- - - CarSim

_ Strong tracking CDKF

(d)

FiguRE 6: Estimated value of the state variables at $120 \mathrm{~km} / \mathrm{h}$ (double lane change road): (a) estimated value of the lateral velocity, (b) estimated value of the lateral acceleration, (c) estimated value of the yaw rate, and (d) estimated value of the side slip angle.

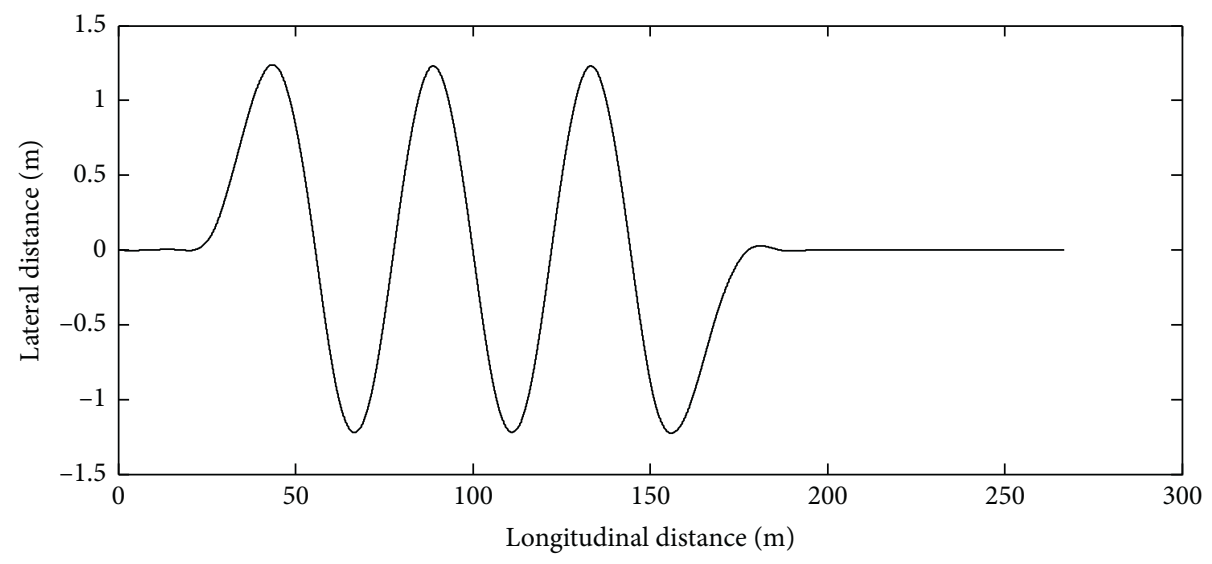

Figure 7: Lateral distance of slalom road at $80 \mathrm{~km} / \mathrm{h}$.

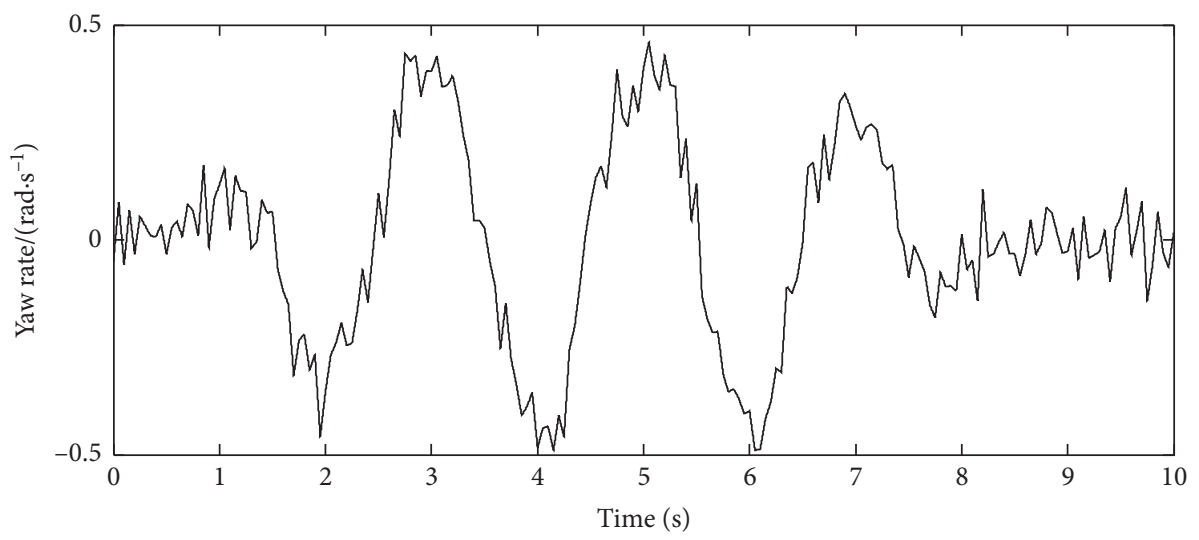

(a)

Figure 8: Continued. 


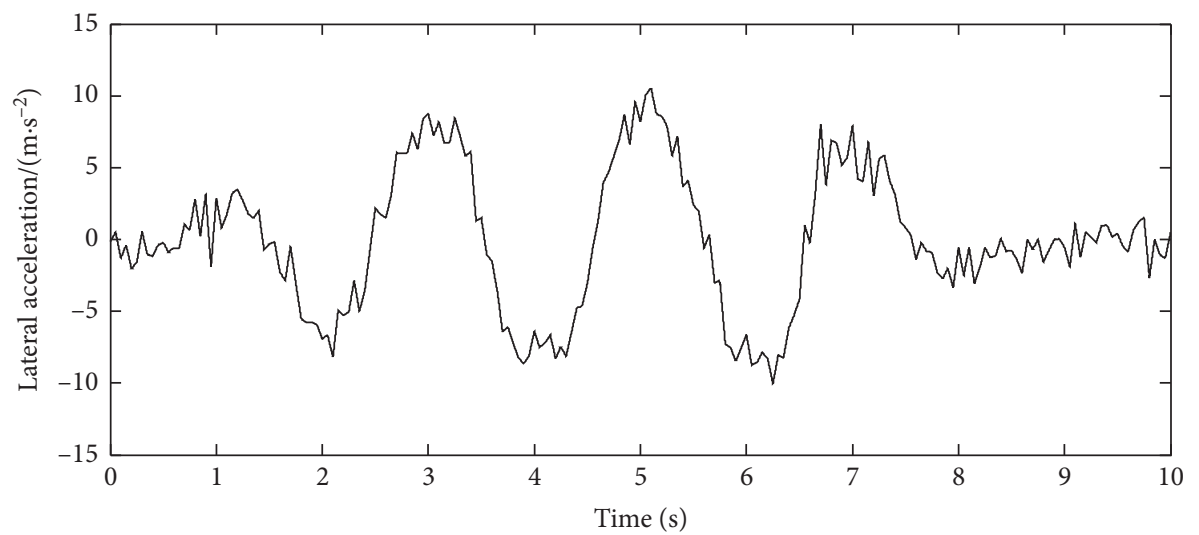

(b)

FIGURE 8: Observational measurements (slalom road): (a) observation measurements of the yaw rate and (b) observational measurements of the lateral acceleration.

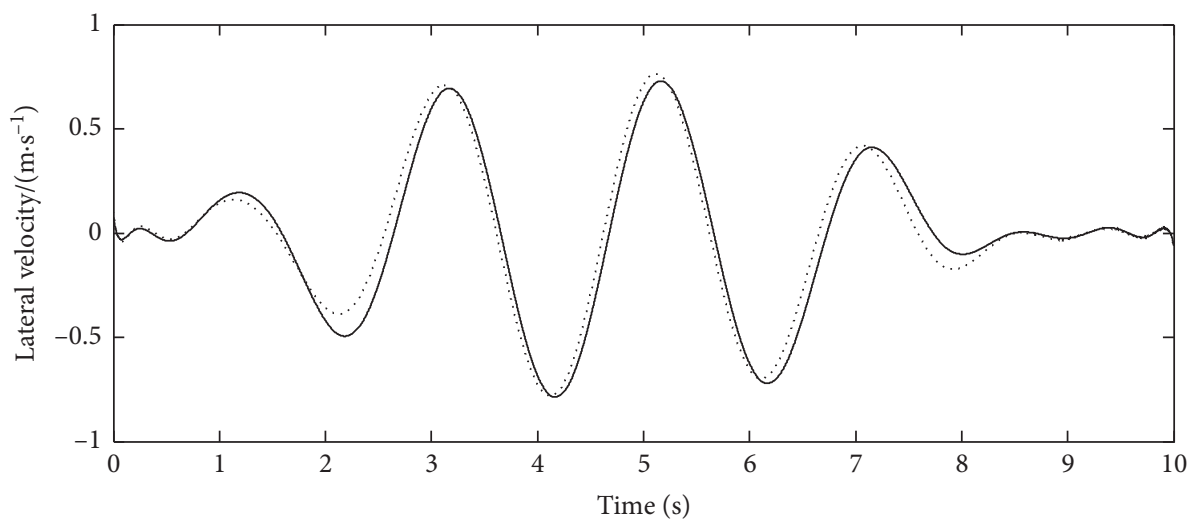

CarSim

Strong tracking CDKF

(a)

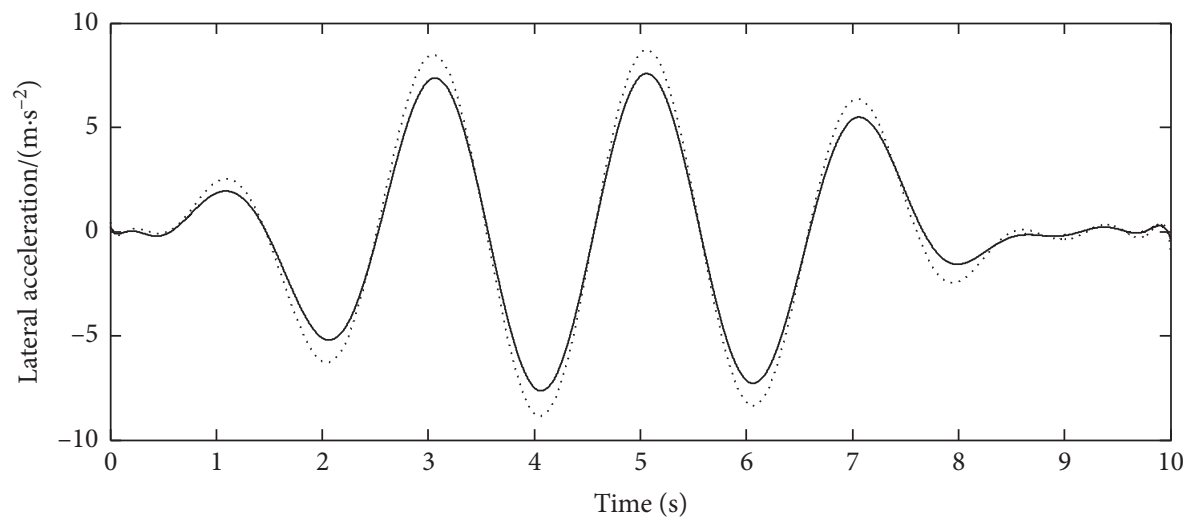

CarSim

Strong tracking CDKF

(b)

Figure 9: Continued. 


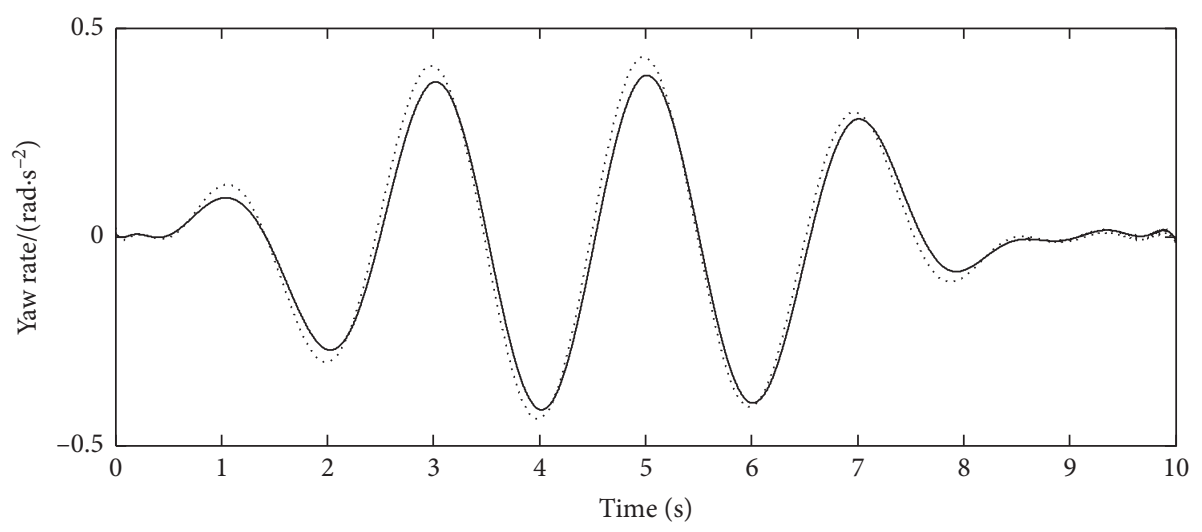

.. CarSim

_ Strong tracking CDKF

(c)

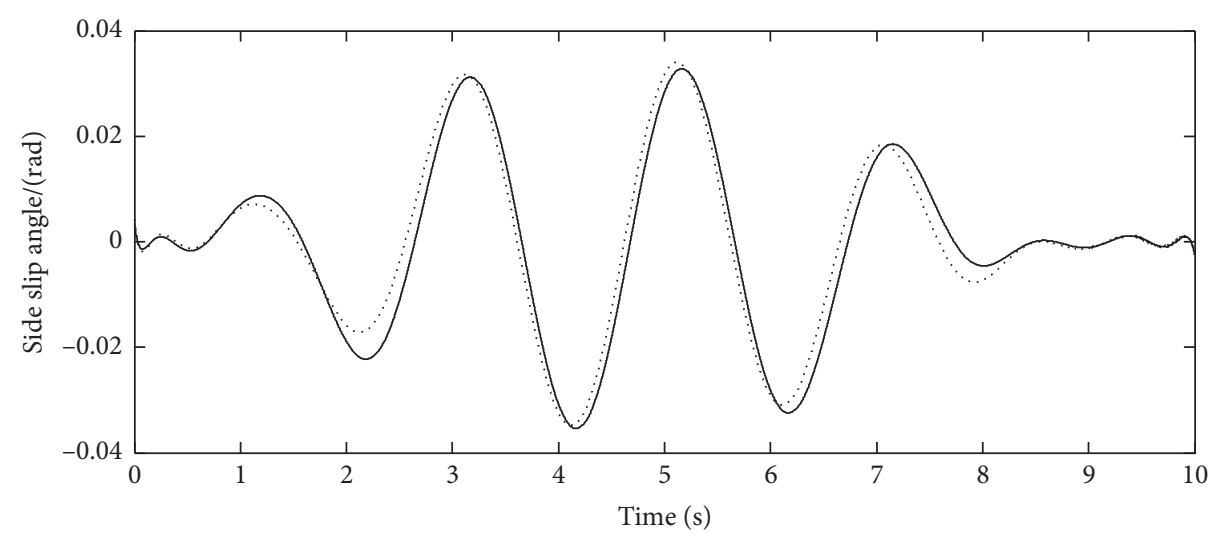

CarSim

__ Strong tracking CDKF

(d)

FIGURE 9: Estimated value of the state variables at $80 \mathrm{~km} / \mathrm{h}$ (slalom road): (a) estimated value of the lateral velocity, (b) estimated value of the lateral acceleration, (c) estimated value of the yaw rate, and (d) estimated value of the side slip angle.

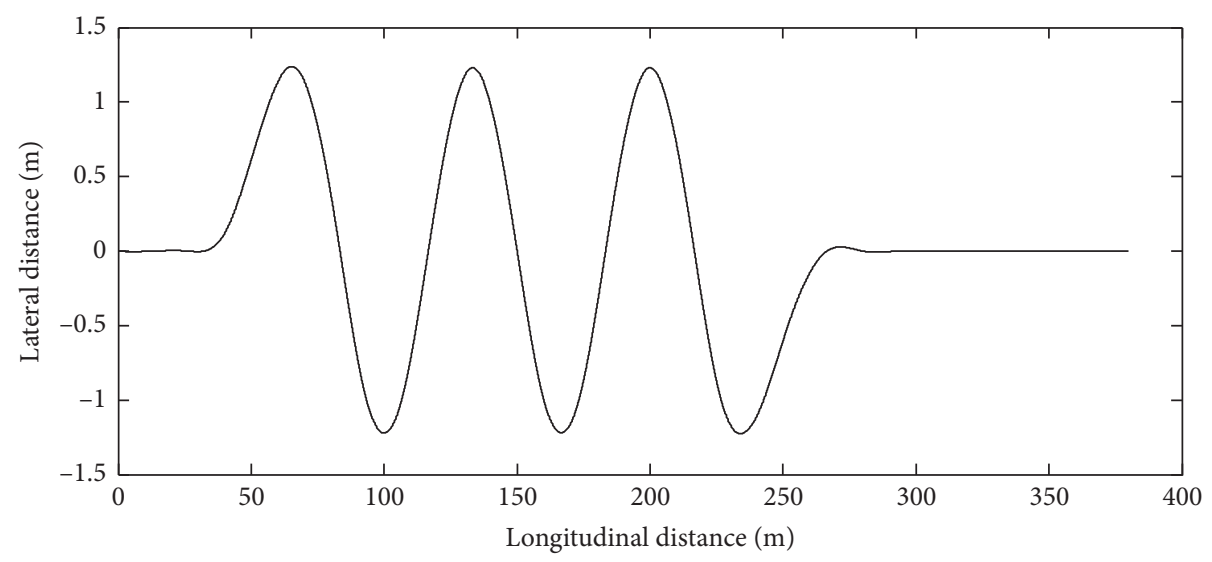

FIGURE 10: Lateral distance of slalom road at $120 \mathrm{~km} / \mathrm{h}$.

the related test data. The test vehicle speed is set as $80 \mathrm{~km} / \mathrm{h}$. An angular rate gyroscope is installed on the vehicle to collect the yaw angular velocity and lateral acceleration of the vehicle in real time. A RACELOGIC VBOX speed sensor is used to measure the longitudinal and lateral speed of the vehicle. A steering torque/angle tester which accuracy is $1 \%$ 


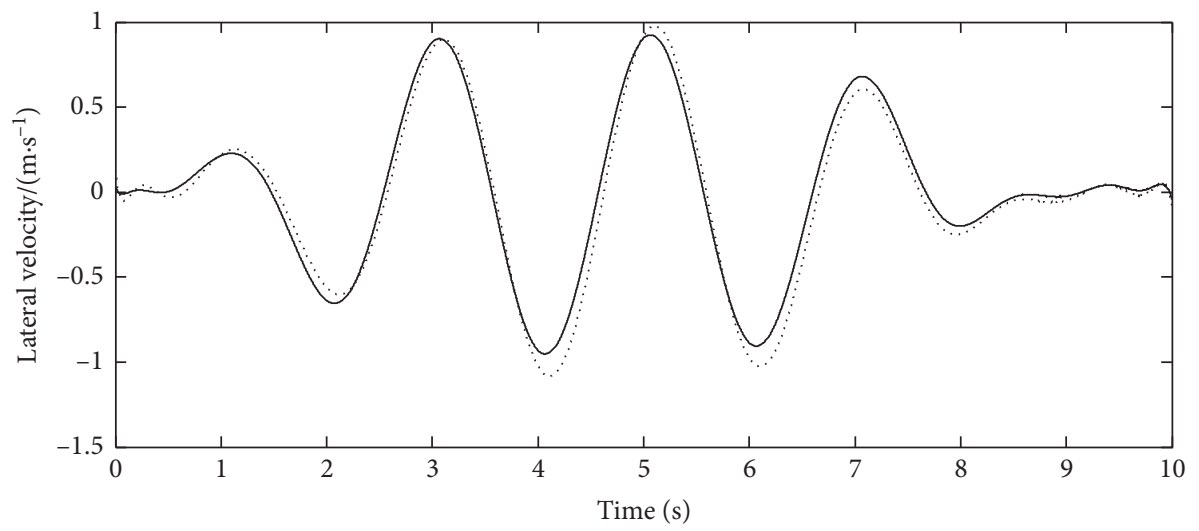

.... CarSim

— Strong tracking CDKF

(a)

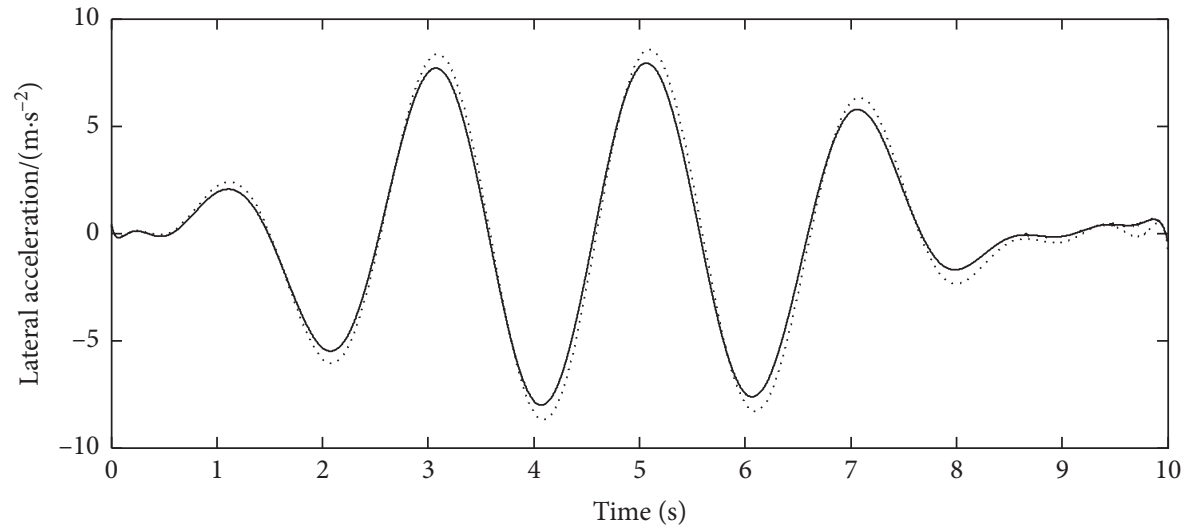

CarSim

Strong tracking CDKF

(b)

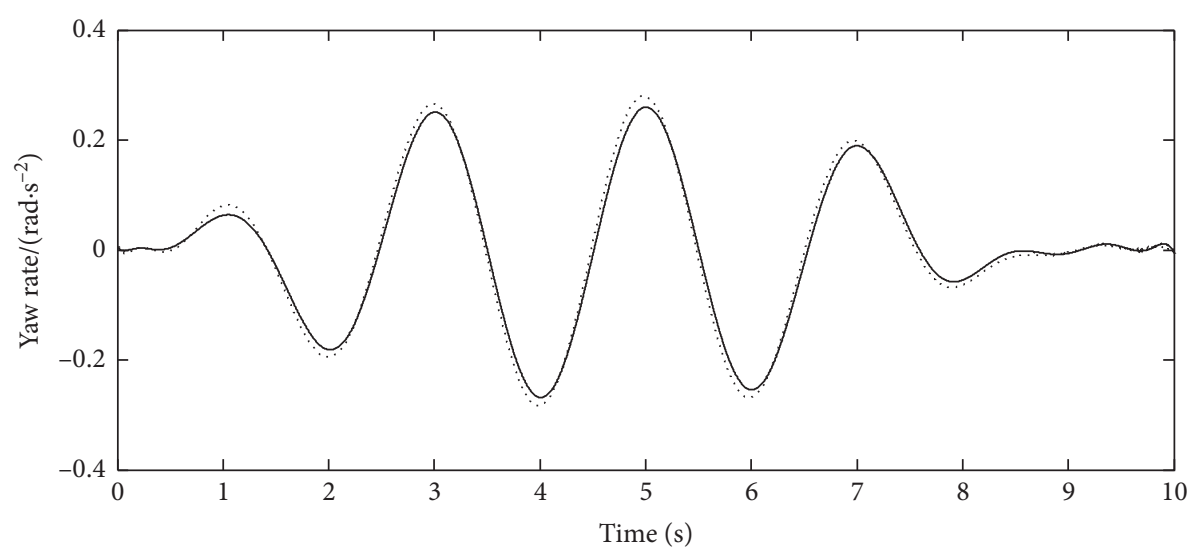

CarSim

Strong tracking CDKF

(c)

FIgURE 11: Continued. 


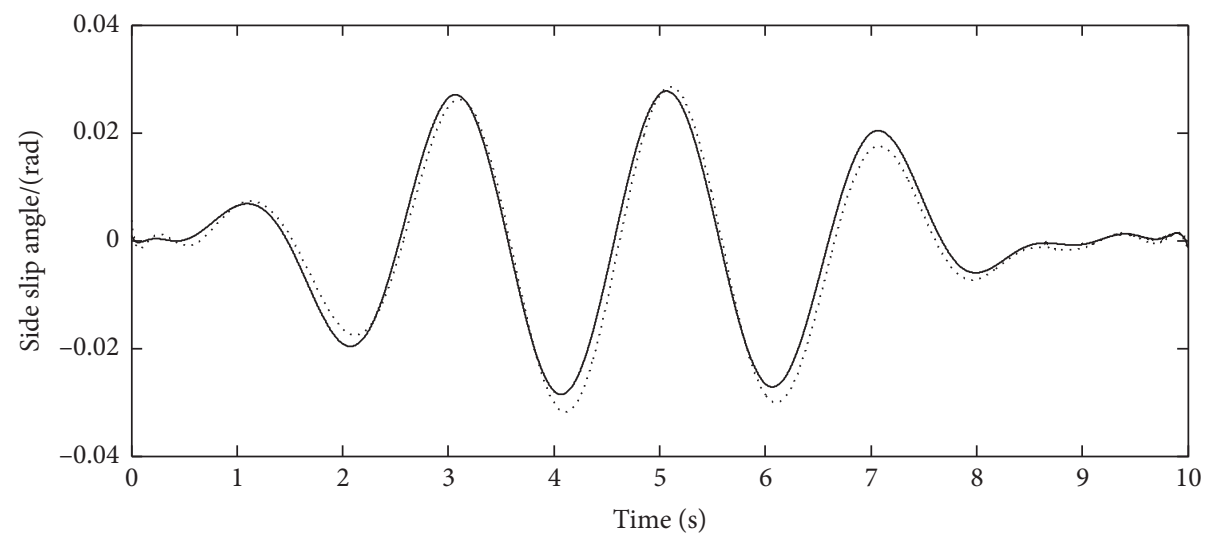

CarSim

Strong tracking $\mathrm{CDKF}$

(d)

FIGURE 11: Estimated value of the state variables at $120 \mathrm{~km} / \mathrm{h}$ (slalom road): (a) estimated value of the lateral velocity, (b) estimated value of the lateral acceleration, (c) estimated value of the yaw rate, and (d) estimated value of the side slip angle.

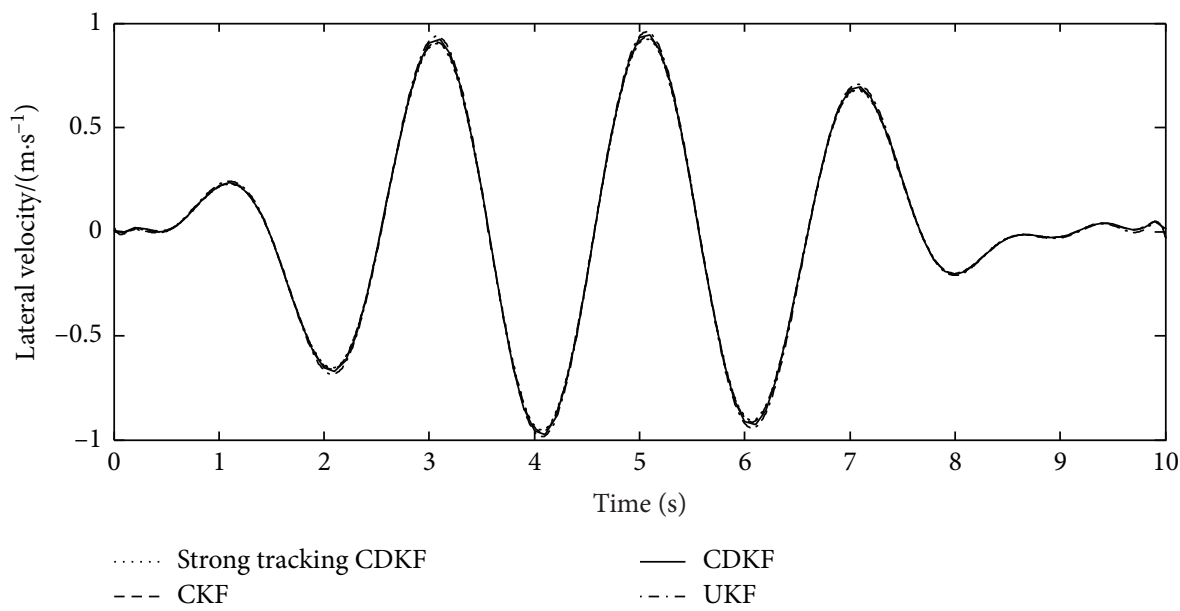

(a)

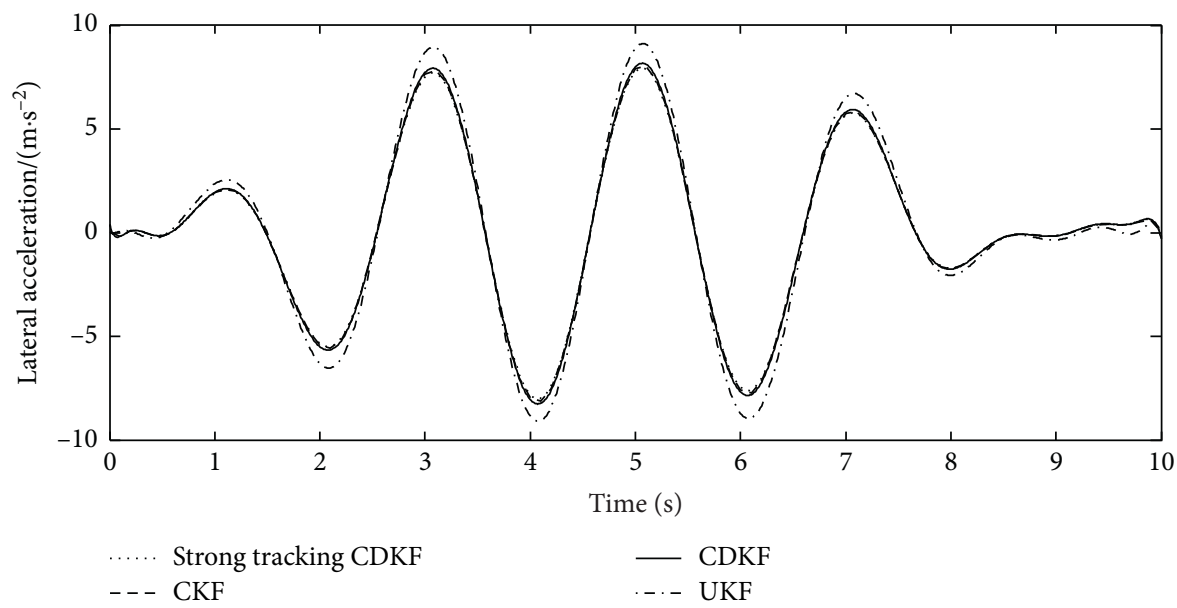

(b)

Figure 12: Continued. 


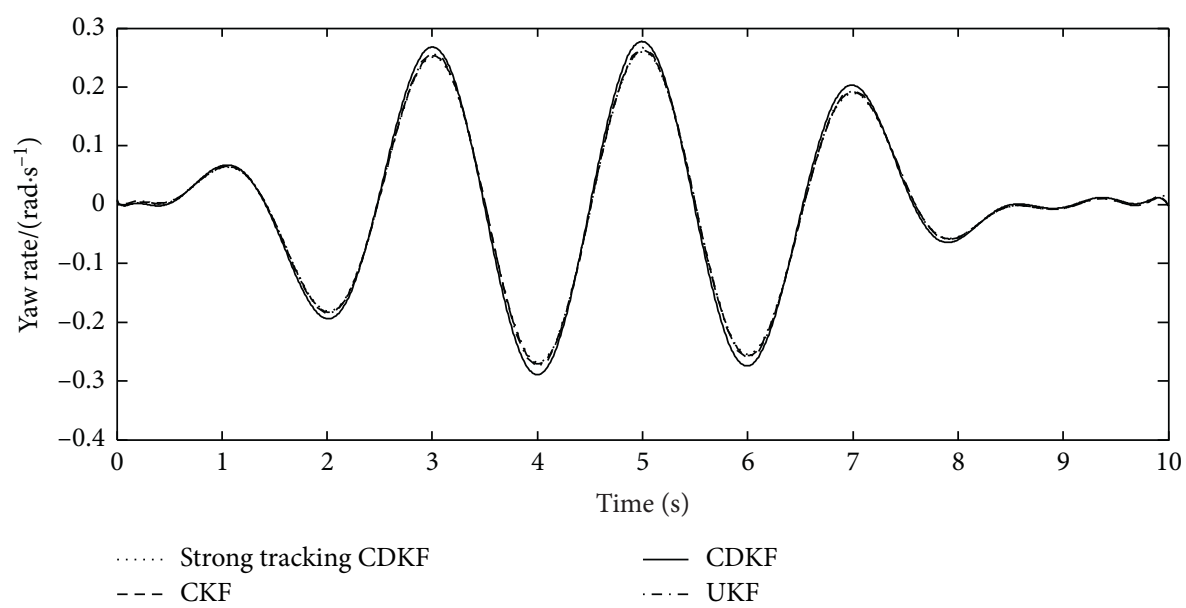

(c)

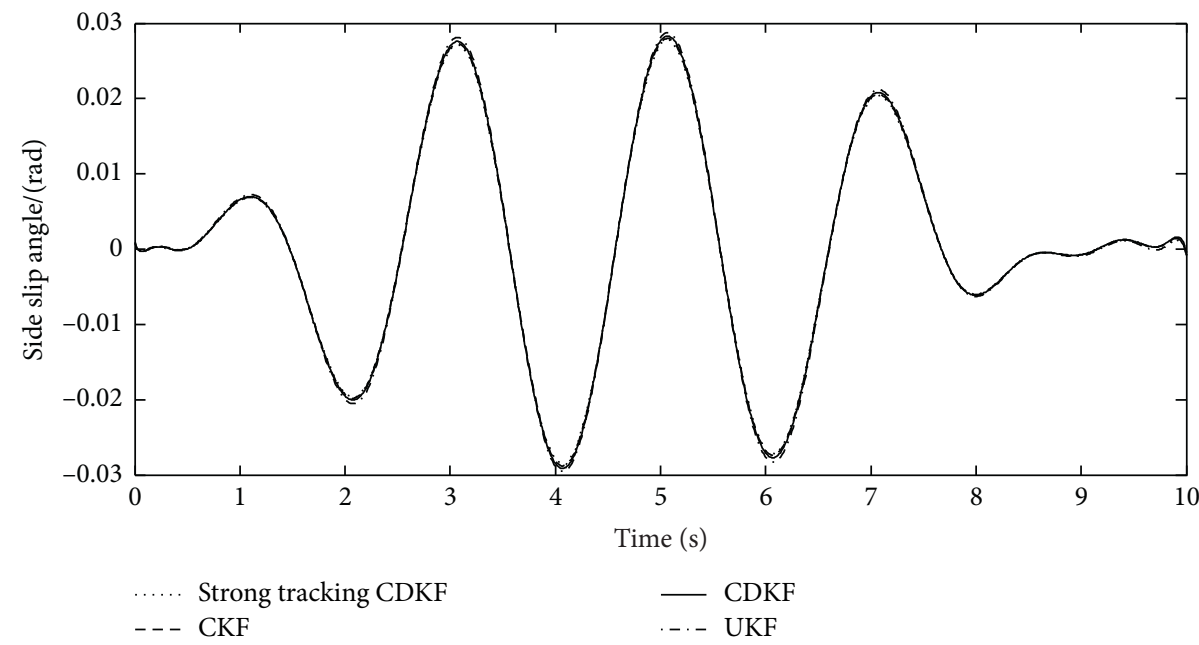

(d)

FIGURE 12: Comparison value of the state variables with different algorithms at $120 \mathrm{~km} / \mathrm{h}$ : (a) estimated value of the lateral velocity, (b) estimated value of the lateral acceleration, (c) estimated value of the yaw rate, and (d) estimated value of the side slip angle.

TABLE 2: Error comparison between the strong tracking CDKF algorithm, CKF, CDKF, and the UKF.

\begin{tabular}{llcccc}
\hline & & Strong tracking CDKF & CKF & CDKF & 0.0647 \\
\hline \multirow{3}{*}{ MAE } & $v(\mathrm{~m} / \mathrm{s})$ & 0.0634 & 0.0638 & 0.0659 \\
& $a_{y}\left(\mathrm{~m} / \mathrm{s}^{2}\right)$ & 0.459 & 0.476 & 0.492 & 0.547 \\
& $r(\mathrm{rad} / \mathrm{s})$ & 0.0163 & 0.0166 & 0.0219 & 0.0221 \\
& $\beta(\mathrm{rad})$ & 0.0019 & 0.0022 & 0.0023 \\
$\mathrm{R}$ & $v(\mathrm{~m} / \mathrm{s})$ & 0.119 & 0.120 & 0.121 & 0.66 \\
& $a_{y}\left(\mathrm{~m} / \mathrm{s}^{2}\right)$ & 0.67 & 0.0271 & 0.0280 & 0.65 \\
& $r(\mathrm{rad} / \mathrm{s})$ & 0.0262 & 0.00340 & 0.00341 & 0.0291 \\
& $\beta(\mathrm{rad})$ & 0.00339 & & & 0.00344 \\
\hline
\end{tabular}

is used for measuring the steering wheel angle. The aforementioned equipments are shown in Figure 13. And the real test vehicle is shown in Figure 14.

Figure 15 shows the result of the steering wheel angle with time measured by the test vehicle with speed of $80 \mathrm{~km} / \mathrm{h}$.

The test estimated values of the four key states are shown in Figure 16(a) to 16(d). From Figures 16(a) to 16(d), it can be seen that the estimated values are basically consistent with the experimental values in the trend, which verifies the effectiveness of the algorithm proposed in this paper for vehicle state estimation. However, there is a certain error between the estimated value and the virtual experimental value. This is because the Fiala tire model used in this paper has a certain deviation when simulating the mechanical characteristics of real vehicle tires. In addition, the measurement error and installation position of the sensors are also important reasons for the deviation between the estimated value and the test value. 


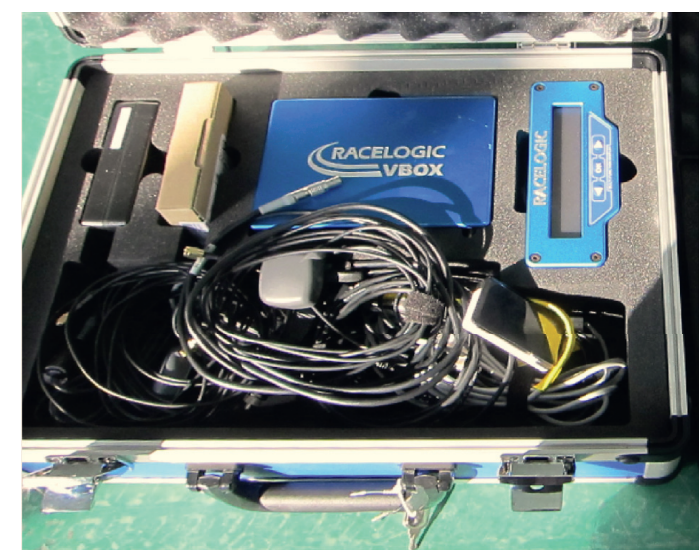

(a)

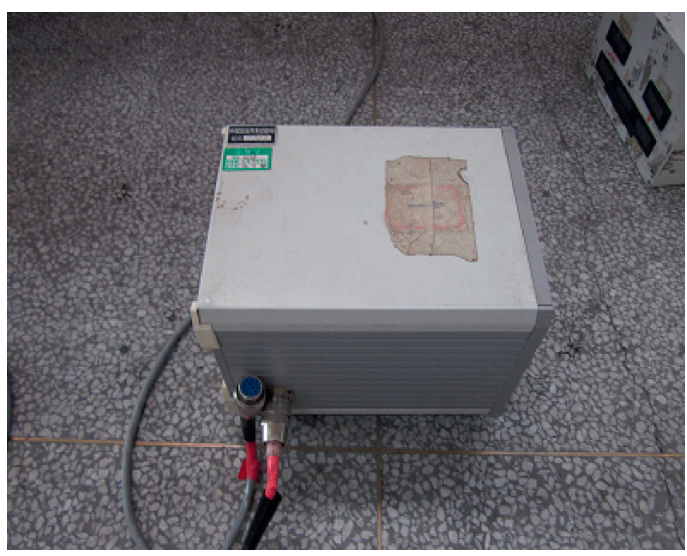

(c)

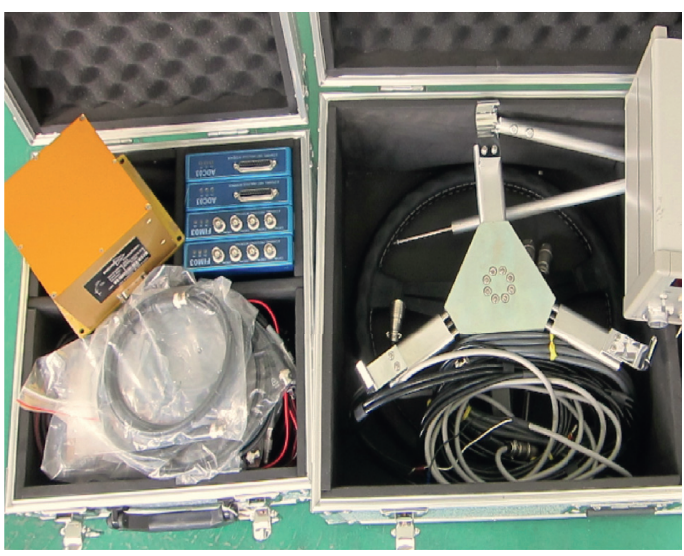

(b)

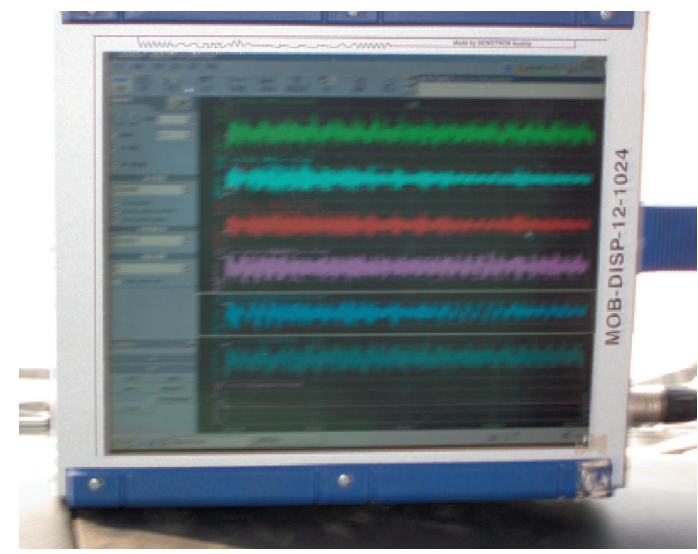

(d)

Figure 13: Measurement equipments: (a) RACELOGIC VBOX speed instrument, (b) steering torque/angle tester, (c) angular rate gyroscope, and (d) DEWESoft digital signal acquisition.
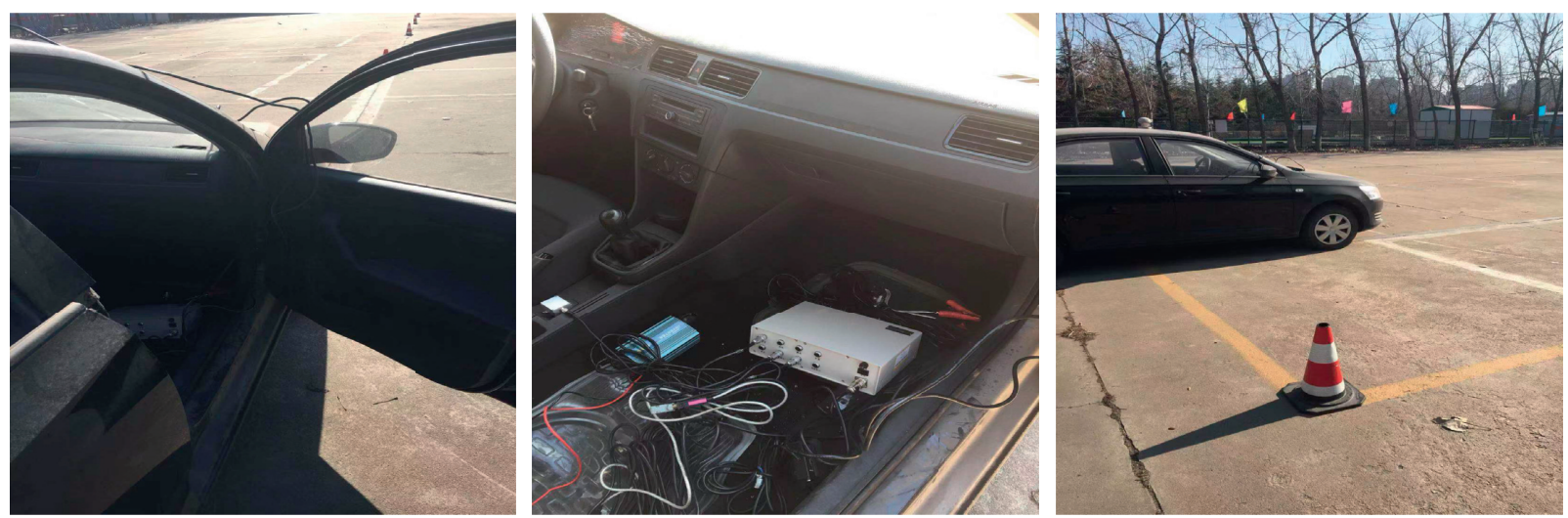

Figure 14: The real test vehicle. 


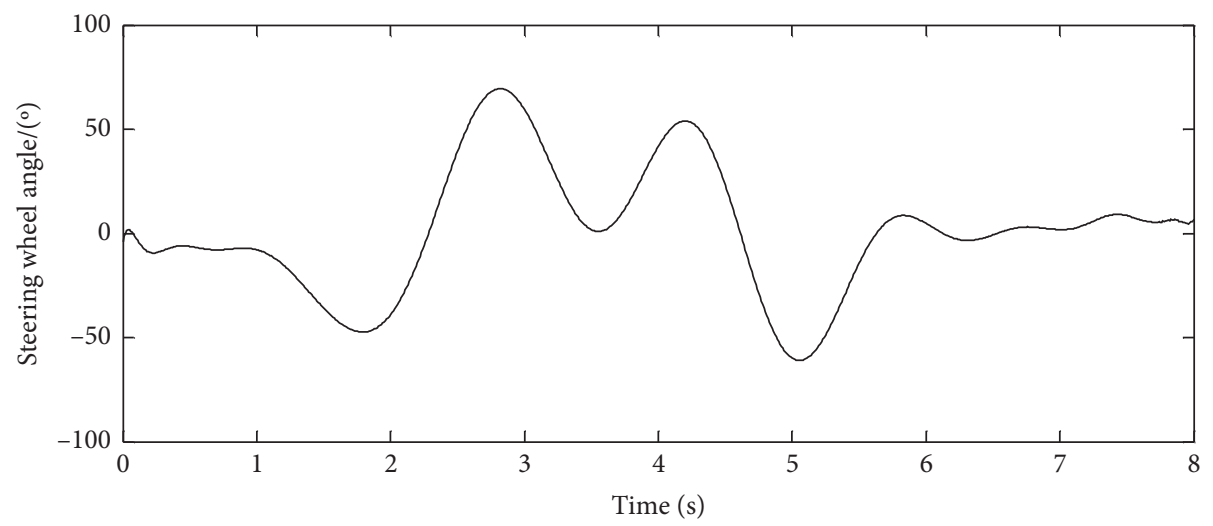

FIGURE 15: Steering wheel angle.

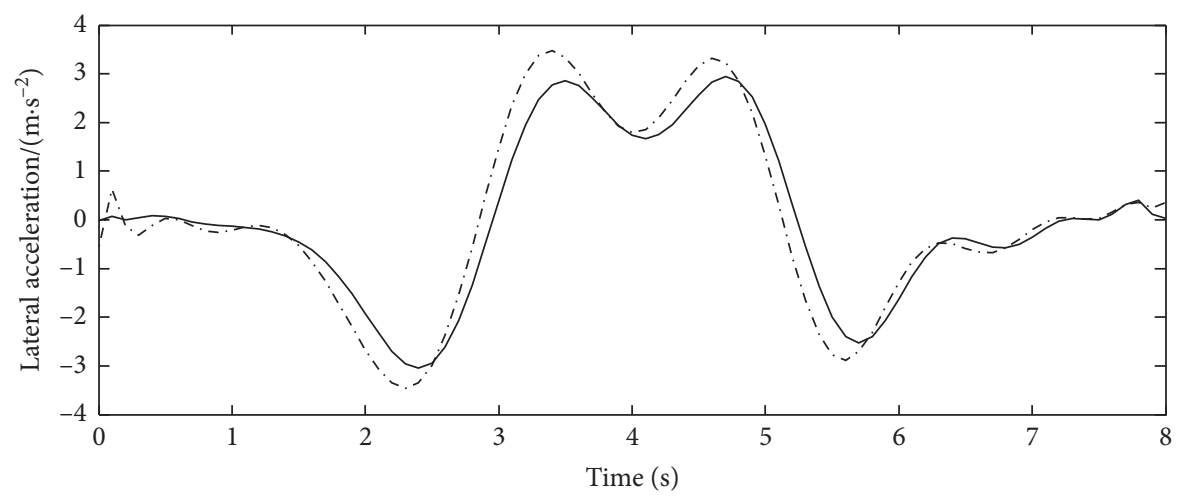

... Test value

_ Strong tracking CDKF

(a)

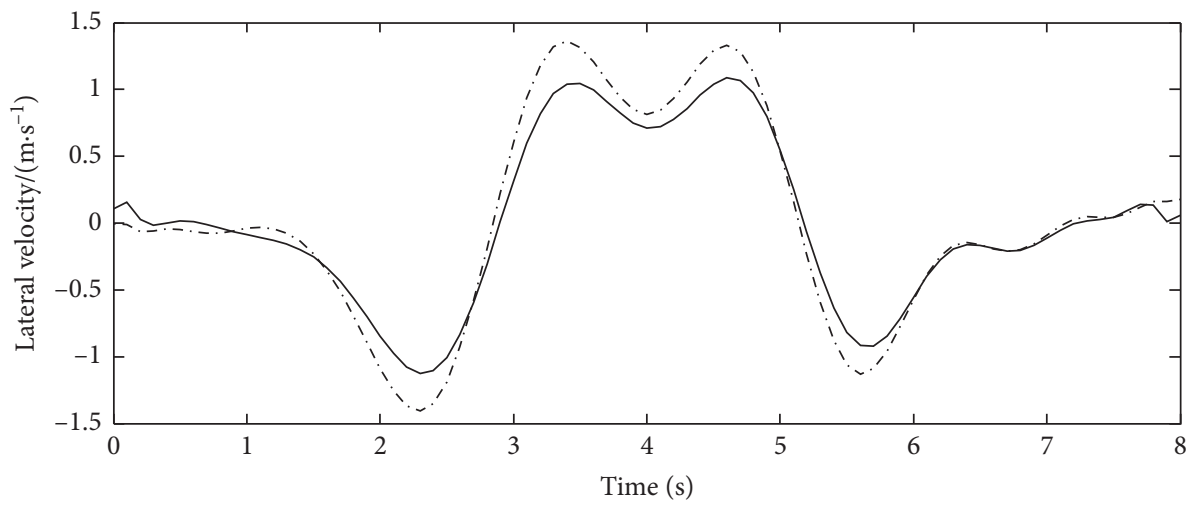

-. - Test value

— Strong tracking CDKF

(b)

Figure 16: Continued. 


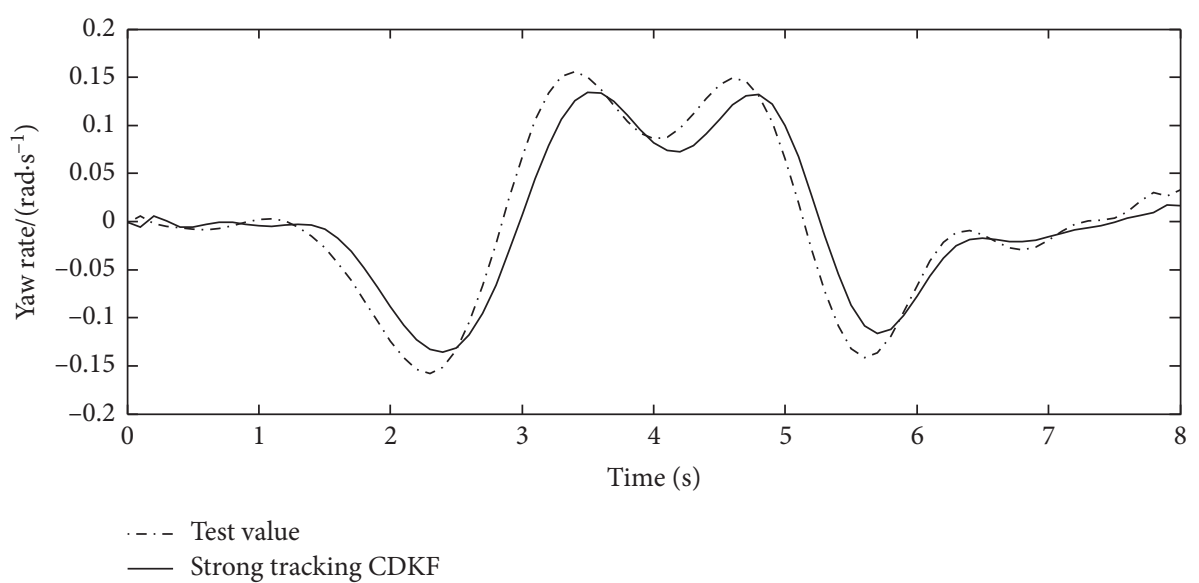

(c)

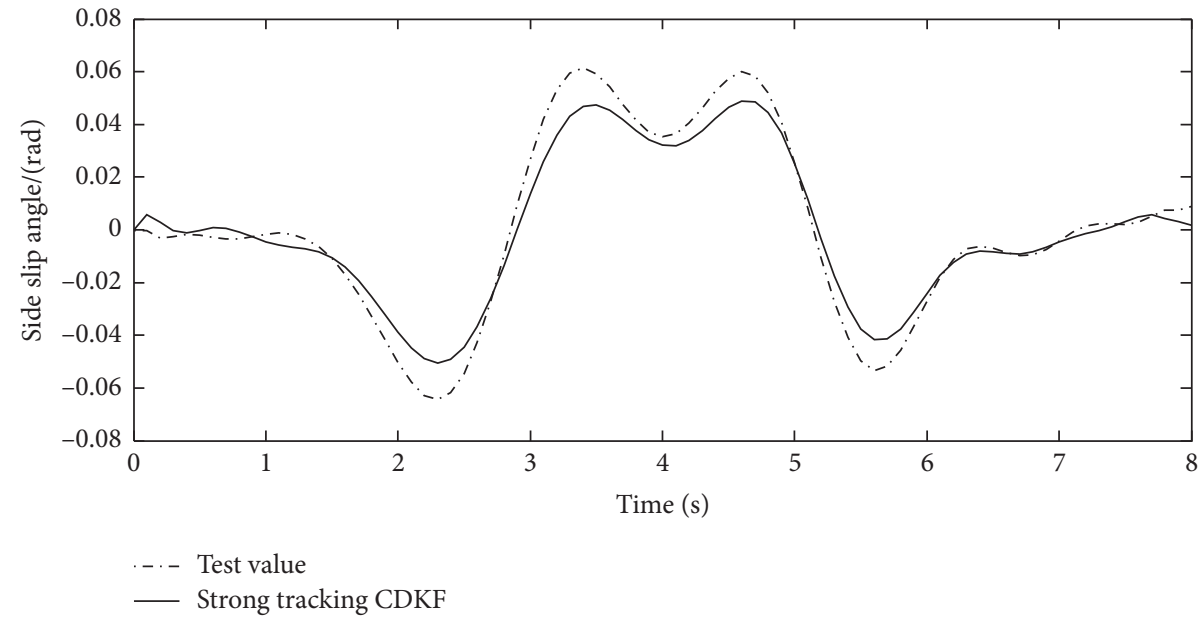

(d)

Figure 16: Comparison of estimation and test value: (a) test and estimated values of the lateral acceleration including measurement noise, (b) test and estimated values of the lateral velocity including measurement noise, (c) test and estimated values of the yaw rate, and (d) test and estimated values of the side slip angle.

\section{Conclusion}

A 3-DOF vehicle state estimation model and a modified Fiala model are established for the problem of vehicle state estimation. And a vehicle state estimation algorithm combined with fuzzy control and Kalman filter is designed based on the strong tracking CDKF theory. The proposed algorithm can adaptively adjust $R$ to perform more accurate and effective vehicle state estimation. Comparing result with the UKF algorithm shows that the strong tracking CDKF algorithm is more accurate and effective than the unscented Kalman filter algorithm to estimate vehicle state parameters. And also the simulation results and the real vehicle test verification indicate that the strong tracking CDKF proposed in this paper can improve the accuracy of vehicle state estimation.

It is believed that in the future the idea of applying the strong tracking CDKF algorithm obtaining accurate and real-time status information of the vehicle during driving to the estimation of key states of the vehicle can provide certain theoretical guidance for the software design of the estimator and automotive dynamic control systems in the vehicle dynamic control system.

\section{Data Availability}

The related data used to support the findings of this study are available from the corresponding author upon request.

\section{Conflicts of Interest}

The authors declare that there are no conflicts of interest regarding the publication of this study.

\section{Acknowledgments}

This research was supported by the Science and Technology Program Foundation of Weifang under Grant 2015GX007. The first author gratefully acknowledges the support agency. 


\section{References}

[1] R. Sakthivel, S. Mohanapriya, B. Kaviarasan et al., "Nonfragile control design and state estimation for vehicle dynamics subject to input delay and actuator faults," The Institution of Engineering and Technology, vol. 14, pp. 134-144, 2019.

[2] A. J. Hamze and G. Ali, "Development of a novel nonlinear estimator based on state-dependent Riccati equation technique for articulated vehicles," Proceedings of the Institution of Mechanical Engineers, Part K: Journal of Multi-Body Dynamics, vol. 233, pp. 516-530, 2019.

[3] L. Chen, X. Yang, J. Z. Zhang et al., "Levenberg-marquardt backpropagation training of multilayer neural networks for state estimation of a safety-critical cyber-physical system," IEEE Transactions on Industrial Informatics, vol. 14, pp. 3436-3446, 2018.

[4] O. Töry, T. Bécsi, S. Aradi et al., "Sensitivity and performance evaluation of multiple-model state estimation algorithms for autonomous vehicle functions," Journal of Advanced Transportation, vol. 1013 pages, 2016.

[5] S. Antonov, A. Fehn, and A. Kugi, "Unscented Kalman filter for vehicle state estimation," Vehicle System Dynamics, vol. 49, no. 9, pp. 1497-1520, 2011.

[6] Y. F. Wang, Z. S. Zhou, C. F. Wei et al., "Host-Target vehicle model-based lateral state estimation for preceding target vehicles considering measurement delay," IEEE Transactions on Industrial Informatics, vol. 14, no. 9, pp. 4190-4199, 2018.

[7] Y. C. Lin and L. Ha, "Adaptive neuro-fuzzy predictor-based control for cooperative adaptive cruise control system," IEEE Transactions on Intelligent Transportation Systems, vol. 65, pp. 1-10, 2019.

[8] C. L. Robert, C. M. John, W. B. Randal et al., "Quadrotors and accelerometers: state estimation with an improved dynamic model," IEEE Control Systems Magazine, vol. 34, pp. 28-41, 2014.

[9] X. B. Han, M. G. Ouyang, L. G. Lu et al., "Simplification of physics-based electrochemical model for lithium ion battery on electric vehicle," Part II: Pseudo-two-dimensional model simplification and state of charge estimation. Journal of Power Sources, vol. 278, pp. 814-825, 2015.

[10] B. Kim, K. Yi, H. J. Yoo et al., “An IMM/EKF approach for enhanced multitarget state estimation for application to integrated risk management system," IEEE Transactions on Vehicular Technology, vol. 64, pp. 876-889, 2015.

[11] G. Reina, M. Paiano, and J.-L. Blanco-Claraco, "Vehicle parameter estimation using a model-based estimator," $M e$ chanical Systems and Signal Processing, vol. 87, pp. 227-241, 2017.

[12] H. Vincent, X. Zhu, and W. Dong, "A two-task hierarchical constrained tri-objective optimization approach for vehicle state estimation under non-gaussian environment," Journal of Computational and Theoretical Nanoscience, vol. 12, pp. 5504-5516, 2015.

[13] H. Ehsan, K. Saeid, K. Amir et al., "Longitudinal vehicle state estimation using nonlinear and parameter-varying observers," Mechatronics, vol. 43, pp. 28-39, 2017.

[14] M. D. Noor-A-Rahim, M. O. Khyam, G. G. Md et al., "Reliable state estimation of an unmanned aerial vehicle over a distributed wireless IoT network," IEEE Transactions on Reliability, vol. 68, pp. 1061-1069, 2019.

[15] K. B. Singh, M. A. Arat, and S. Taheri, "Literature review and fundamental approaches for vehicle and tire state estimation,"
International Journal of Vehicle Mechanics and Mobility, vol. 57, pp. 1643-1665, 2019.

[16] P. Harry, J. M. Benjamin, and M. Christian, “Towards a model based sensor measurement variance input for extended Kalman filter state estimation," Drone, vol. 3, pp. 1-18, 2019.

[17] A. J. Hamze and G. Ali, "Nonlinear estimator design based on extended Kalman filter approach for state estimation of articulated heavy vehicle," Proceedings of the Institution of Mechanical Engineers, Part K: Journal of Multi-body Dynamics, vol. 233, pp. 254-265, 2019.

[18] Y. Qin, Z. Wang, C. Xiang, M. Dong, C. Hu, and R. Wang, "A novel global sensitivity analysis on the observation accuracy of the coupled vehicle model," Vehicle System Dynamics, vol. 57, no. 10, pp. 1445-1466, 2019.

[19] A. Afzal, A. A. N. Syed, and W. David, "Real-time dynamic traffic control based on traffic-state estimation," Transportation Research Record: Journal of the Transportation Research Board, vol. 2673, pp. 584-595, 2019.

[20] S. Wang, Q. Yu, X. Zhao, S. Zhang, and Y. Ye, "Vehicle sideslip angle estimation based on SVD-UPF algorithm," Journal of Intelligent \& Fuzzy Systems, vol. 37, no. 4, pp. 4563-4573, 2019.

[21] H. Liu, J. Yang, H. Yang, and F. Yi, "Soft sensor of vehicle state estimation based on the Kernel principal component and improved neural network," Journal of Sensors, vol. 2016, Article ID 9568785, 8 pages, 2016.

[22] W. Alexander, S. Tim, B. Johsnnes et al., "Vehicle dynamics state estimation and localization for high performance race cars," IFACA, vol. 52, pp. 154-161, 2019.

[23] B. B. Gao, G. G. Hu, Y. M. Zhong et al., "Cubature rule-based distributed optimal fusion with identification and prediction of kinematic model error for integrated UAV navigation," Aerospace Science and Technology, vol. 109, pp. 1-21, 2021.

[24] B. Gao, G. Hu, Y. Zhong, and X. Zhu, "Cubature Kalman filter with both adaptability and robustness for tightly-coupled GNSS/INS integration," IEEE Sensors Journal, vol. 21, no. 13, pp. 14997-15011, 2021.

[25] Y. J. Liu and C. H. Dou, "Vehicle state estimation based on unscented kalman filtering and a genetic algorithm," $S A E$ International Journal of Commercial Vehicles, vol. 14, pp. 1-15, 2020.

[26] M. Norgarrd, N. K. Poulsen, and O. Ravn, "New developments in state estimation for nonlinear systems," Automatica, vol. 36, no. 11, pp. 1627-1638, 2000.

[27] Y. J. Liu, C. H. Dou, F. P. Shen et al., "Vehicle state estimation based on unscented kalman filtering and a genetic-particle swarm algorithm," Journal of The Institution of Engineers (India): Series C, vol. 103, pp. 1-23, 2021. 